\title{
Oligothiophene-Naphthalimide Hybrids Connected through Rigid and Conjugated Linkers in Organic Electronics: An Overview
}

\author{
Matías J. Alonso-Navarro ${ }^{1,2,+} \mathbb{D}$, Elena Gala ${ }^{1,2,+} \mathbb{D}$, M. Mar Ramos ${ }^{2} \mathbb{D}$, Rocío Ponce Ortiz ${ }^{3, *(\mathbb{D})}$ \\ and José L. Segura $1, *(\mathbb{D}$
}

1 Department of Organic Chemistry, Faculty of Chemistry, Complutense University of Madrid, 28040 Madrid, Spain; matiasjesus.alonso@urjc.es (M.J.A.-N.); elena.gala@urjc.es (E.G.)

2 Chemical and Environmental Technology Department, Universidad Rey Juan Carlos, 28933 Móstoles, Spain; mariamar.ramos@urjc.es

3 Department of Physical Chemistry, University of Málaga, 29071 Málaga, Spain

* Correspondence: rocioponce@uma.es (R.P.O.); segura@ucm.es (J.L.S.); Tel.: +34-952131863 (R.P.O.); +34-913945142 (J.L.S.)

+ These authors contributed equally to this work.

check for updates

Citation: Alonso-Navarro, M.J.; Gala, E.; Ramos, M.M.; Ponce Ortiz, R.; Segura, J.L. Oligothiophene-

Naphthalimide Hybrids Connected through Rigid and Conjugated Linkers in Organic Electronics: An Overview. Electron. Mater. 2021, 2, 222-252. https://doi.org/10.3390/ electronicmat2020017

Academic Editor: Roberto Centore

Received: 1 May 2021

Accepted: 1 June 2021

Published: 5 June 2021

Publisher's Note: MDPI stays neutral with regard to jurisdictional claims in published maps and institutional affiliations.

Copyright: (c) 2021 by the authors. Licensee MDPI, Basel, Switzerland. This article is an open access article distributed under the terms and conditions of the Creative Commons Attribution (CC BY) license (https:/ / creativecommons.org/licenses/by/ $4.0 /)$.
Abstract: In this article, we summarize the synthetic approaches developed in our research groups during the last decade to efficiently tune the optical, electrochemical and morphological characteristics of oligothiophene-naphthalimide assemblies. Different variables were tuned in these organic semiconductors, such as the planarity and the length of their $\pi$-conjugated backbones, the topology and energy levels of the frontier molecular orbitals (HOMO and LUMO) and their molecular dipole moments. The tuning of these properties can be connected with the microstructure properties observed by atomic force microscopy (AFM) and X-ray diffraction (XRD) in thin films as well as with the performances in organic field-effect transistors (OFETs). The possibility of incorporating these donor-acceptor assemblies into macromolecular structures is also addressed, and some innovative applications for these macromolecular systems, such as the degradation of organic pollutants in aqueous media, are also presented.

Keywords: organic electronics; oligothiophene-naphthalimide; semiconductor; frontier molecular orbitals; ambipolarity; thin films; organic field-effect transistors; degradation of contaminants

\section{Introduction}

$\pi$-conjugated small molecules and polymers are extensively used in organic electronics because of their low-cost manufacturing, light weight, good processability and good ability to be deposited on flexible substrates [1,2] in comparison with conventional inorganic semiconductors $[3,4]$. In order to efficiently tune the charge transport abilities and electronic performances of $\pi$-conjugated materials in (opto)electronic devices, an indepth understanding of their molecular structures, supramolecular organization and film morphology are required. Among the most extensively investigated electron accepting organic materials, naphthalenediimides (NDI) [5-7] and perylenediimides (PDI) [1,8] stand out because of their good electron-withdrawing abilities and their low-lying LUMO levels, which properly stabilize multiple negative charges. On the other hand, thiophene-based $\pi$-conjugated oligomers and polymers are efficient electron-donor systems with tunable HOMO levels, suitable to stabilize multiple positive charges.

For these reasons, donor-acceptor assemblies based on oligothiophene and naphthalimide moieties have received a great deal of attention in organic electronics $[1,4,9]$. In this framework, during the last few years, we have been active in the syntheses of oligothiophene-naphthalimide assemblies in which the donor and acceptor electroactive units are connected through rigid heterocycles. The connection of flat and rigid donor and 
acceptor moieties through rigid heterocyclic spacers is an efficient strategy to minimize skeletal distortions, allowing closer intermolecular $\pi-\pi$ stacking and extended intramolecular $\pi$ conjugation. Furthermore, through the suitable functionalization of these innovative organic semiconductors, they can be designed to exhibit a good film forming ability and low reorganization energies for both electron and hole transports.

Therefore, in this feature article, we summarize our contribution to the development of synthetic strategies toward several families of ambipolar organic electronic materials based on oligothiophene and 1,8-naphthalimide units connected via different rigid heterocyclic linkers. We highlight the principles of design of the different families of oligothiophene-naphthalimide assemblies and rationalize how the synthetic modifications affect the molecular geometry, the frontier energy levels, the thin-film microstructures and their performances in organic field-effect transistors (OFETs).

To evaluate the charge-transport characteristics of all the systems reviewed in this work, top-contact/bottom-gate thin-film transistors were fabricated by either vapor or solution deposition of the semiconducting films onto octadecyltrichlorosilane (OTS)functionalized $\mathrm{Si} / \mathrm{SiO}_{2}$ substrates and/or onto hexamethyldisilazane (HMDS)-treated $\mathrm{Si} / \mathrm{SiO}_{2}$ substrates, followed by gold deposition through a shadow mask to define source and drain electrodes, with different channel lengths and widths. The performance parameters were extracted from the I-V response plots, in the saturation regime, by using the assumptions of conventional transistor formalism, Equation (1):

$$
\left(I_{D}\right)_{s a t}=\frac{W}{2 L} \mu C\left(V_{G}-V_{T}\right)^{2}
$$

where $\left(I_{D}\right)_{\text {sat }}$ is the drain current in the saturation regime, $W$ the channel width, $L$ the channel length, $C$ the capacitance per unit area of the insulator layer and $V_{G}$ the gate voltage.

Thus, the linear fitting of the $I_{D}{ }^{1 / 2}$ versus $V_{G}$ plot, in the saturation regime, allowed mobility, $\mu$, and threshold voltage, $V_{T}$, values estimation.

\section{Amidine-Based Oligothiophene-Rylenimide Semiconductors}

As it has been already mentioned, naphthalene and perylene diimide derivatives have been widely used as electron transport materials in a variety of device architectures $[1,8]$. The connection of electron donor oligothiophene units to the rylene skeleton provides an enhancement of the $p$-type behavior of the novel rylene derivative formed, thus enabling ambipolar transport. Our first approach in this direction involves the syntheses of a series of semiconductors based on thiophene-fused rylenimide derivatives via imidazole linkers (Figure 1). This family of oligothiophene-imidazole-rylenimide assemblies was designed with the aim to study the influence of different variables in OFET devices: (i) the effect of the length of the oligothiophene fragment on the charge transport characteristics, (ii) the effect of the replacement of some thiophene units by weaker electron donor benzene moieties and (iii) the effect of the lateral extension of the $\pi$-conjugated systems via naphthalene diimide vs. perylene diimide core interchange.

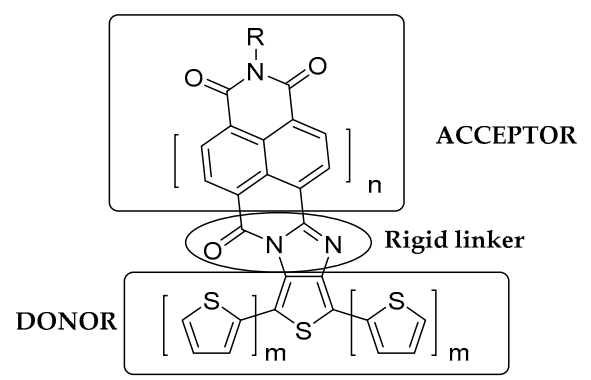

Figure 1. General structure of first generation of thiophene-rylenimide derivatives. 
This first-generation of organic semiconductors based on rylenimide-fused oligothiophene units was obtained by condensation between suitable diaminooligothiophene unit 2 and the corresponding monoanhydride monoimide derivative $\mathbf{1}$ or $\mathbf{3}$ as it is depicted in Scheme 1 [10-12]. An analogue of NDI-3T in which the central thiophene unit is replaced by a benzene moiety, NDI-Ph-T-Ph (Figure 2) was also obtained by following a similar synthetic procedure. Different alkyl chains were introduced at the imide nitrogen of the NDI and PDI derivatives in order to tune the processability of these semiconductors without significantly modifying their photo- and electrochemical properties [13-15].
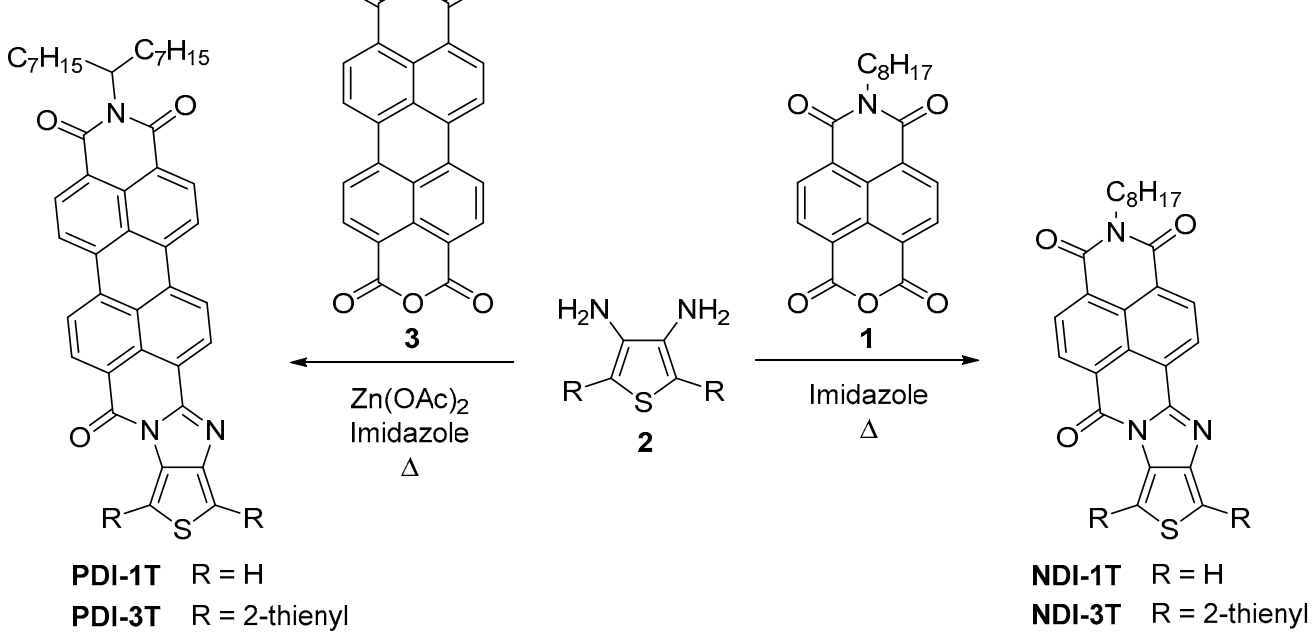

Scheme 1. Synthesis of NDI and PDI derivatives.

The molecular geometries of the oligothiophene-naphthalimide assemblies were optimized by DFT calculations at the B3LYP/6-31G** level. For NDI-3T, PDI-3T and NDIT-Ph-T, it is observed that the end-capped thiophenes close to the keto groups are severely twisted with dihedral angles above $50^{\circ}$ with respect to the molecular plane because of the repulsive interactions between the sulfur atom of the heterocycle unit and the neighboring carbonyl group [12]. Single crystals of NDI-T-Ph-T were grown by slow vapor diffusion of hexane into a chloroform solution, and the molecular structure obtained by X-ray diffraction analyses confirms the twist of the thiophene unit close to the carbonyl group (Figure 2). On the other hand, the systems with a single thiophene unit, NDI-1T and PDI-1T, resulted completely planar because of the absence of nonbonded electronic repulsions.

The UV-vis absorption spectrum of NDI-3T presents three well-defined bands in the 300-600 $\mathrm{nm}$ region, highlighting the broad absorption band around $583 \mathrm{~nm}$ which is ascribed to an intramolecular charge-transfer excitation (ICT) from the HOMO, mainly located into the oligothiophene fragment, to the LUMO, delocalized over the naphthalimide moiety [11]. The perylenimide-based analogue, PDI-3T, shows an enhanced absorption cross section throughout the whole UV-vis spectrum with a strong ICT band at $610 \mathrm{~nm}$ extending up to $800 \mathrm{~nm}$ [10]. It is also worth mentioning that the characteristic fluorescence of rylenimides $[5,16]$ is absent in both NDI-3T and PDI-3T due to the strong charge transfer component. 
<smiles>CCCN1C(=O)c2ccc3c(=O)n4c(nc5c(-c6cccs6)ccc(-c6cccs6)c54)c4ccc(c2c34)C1=O</smiles>

NDI-T-Ph-T

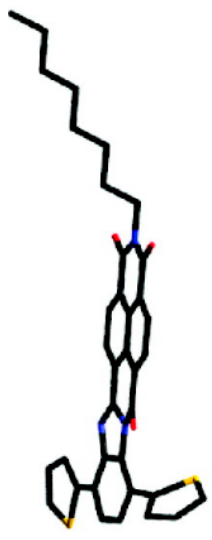

$\theta=56^{\circ}$ (a)

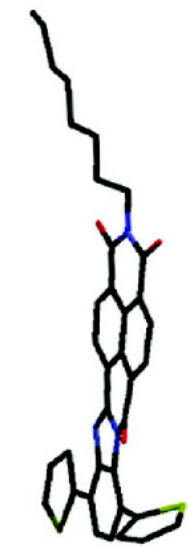

$\theta=64^{\circ}$

(c) (b)

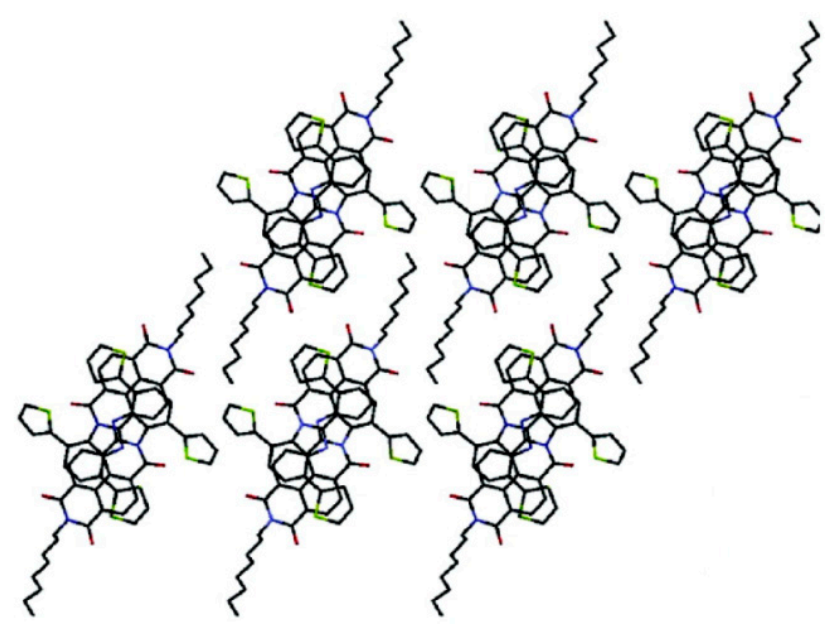

(d)

Figure 2. (a) Molecular structure of NDI-T-Ph-T. (b) Molecular geometry derived from DFT calculations at the B3LYP/6$31 G^{* *}$ level of NDI-T-Ph-T. (c) Molecular geometry derived from X-ray diffraction analysis of NDI-T-Ph-T. $\theta$ indicates the dihedral angle between the external twisted thiophene and the central benzene plane. (d) Molecular structure and packing motif of single crystal of NDI-T-Ph-T, viewed along the $b$-axis. Adapted with permission from J. Am. Chem. Soc. 2010, 132, 24, 8440-8452. Copyright@ 2010 American Chemical Society [12].

Electrochemical characterization of these assemblies was carried out by using cyclic voltammetry measurements. Both reduction and oxidation processes were observed in all the naphthalene and peryleneimide derivatives (Table 1). From these data, LUMO and HOMO energies could be estimated using standard approximations (Figure 3 and Table 1) $[17,18]$, being $-3.79 /-5.83 \mathrm{eV}$ for NDI-1T and $-3.87 /-5.55 \mathrm{eV}$ for the NDI3T analogue. A slight stabilization of the LUMO and a remarkable destabilization of the HOMO energy level around $0.3 \mathrm{eV}$ is observed when the oligothiophene fragment is extended. This electrochemical band gap reduction is consistent with the band gap compression observed in the UV-vis spectra. Further reduction of the electrochemical band gap can be achieved by extending the $\pi$-system of the rylenimide unit through replacement of the naphthalimide moiety by the larger perylenimide analogue. Thus, LUMO/HOMO energies were estimated to be $-3.88 /-5.76 \mathrm{eV}$ for PDI-1T and $-3.89 /-5.55 \mathrm{eV}$ for PDI-3T. The extension of the oligothiophene moiety does not significantly alter the LUMO level, while it produces a destabilization of the HOMO level. 

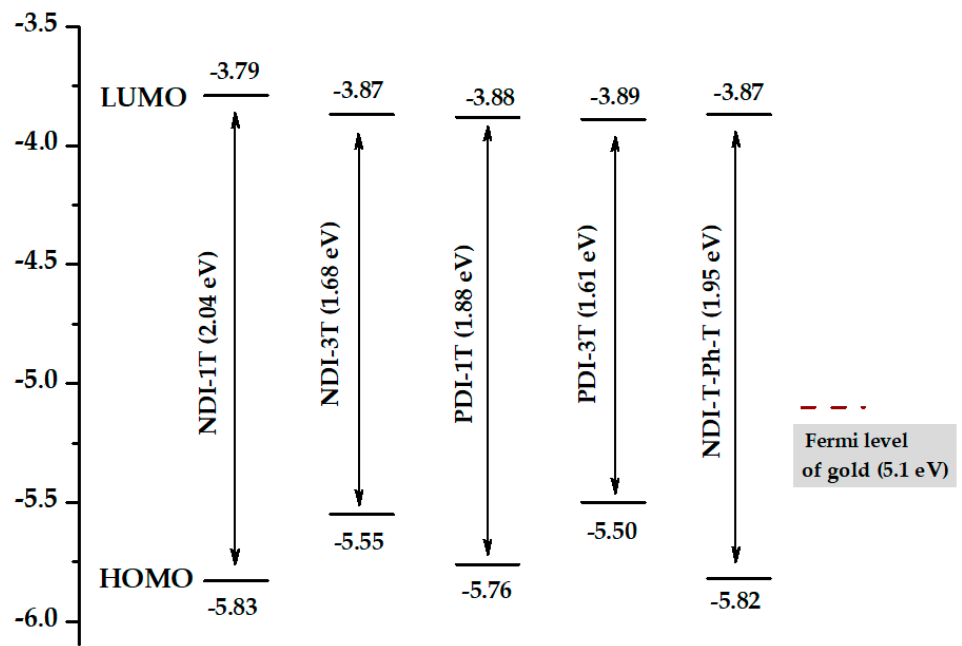

Figure 3. HOMO and LUMO energy levels for NDI-1T, NDI-3T, PDI-1T, PDI-3T and NDI-T-Ph-T semiconductors.

Table 1. Electrochemical potentials versus $\mathrm{SCE}$ in $\mathrm{CH}_{2} \mathrm{Cl}_{2}$ (referenced to $\mathrm{Fc} / \mathrm{Fc}^{+}$couple) of NDI-1T, NDI-3T, PDI-1T, PDI-3T and NDI-T-Ph-T and frontier molecular orbital energy levels estimated from CV data.

\begin{tabular}{cccccccc}
\hline Semiconductor & $E_{\text {red1 }}$ & $E_{\text {red2 }}$ & $E_{\text {ox1 }}$ & $E_{\text {ox2 }}$ & $E_{\mathrm{g}}$ CV & LUMO $^{\mathbf{b}}$ & HOMO $^{\mathbf{c}}$ \\
\hline NDI-1T & -0.65 & -1.04 & $1.39^{\mathrm{a}}$ & - & 2.04 & -3.79 & -5.83 \\
\hline NDI-3T & -0.57 & -0.93 & $1.11^{\mathrm{a}}$ & - & 1.68 & -3.87 & -5.55 \\
\hline PDI-1T & -0.56 & -0.74 & $1.32^{\mathrm{a}}$ & - & 1.88 & -3.88 & -5.76 \\
\hline PDI-3T & -0.55 & -0.71 & $1.06^{\mathrm{a}}$ & - & 1.61 & -3.89 & -5.50 \\
\hline NDI-T-Ph-T & -0.57 & -0.95 & $1.42^{\mathrm{a}}$ & $1.62^{\mathrm{a}}$ & 1.95 & -3.87 & -5.82 \\
\hline
\end{tabular}

${ }^{\mathrm{a}}$ Irreversible. ${ }^{\mathrm{b}} \mathrm{LUMO}$ energy levels estimated vs. vacuum level from $E_{\mathrm{LUMO}}=-4.44 \mathrm{eV}-\mathrm{e} E_{\mathrm{red} 1} \cdot{ }^{\mathrm{c}}$ Estimated from $\mathrm{HOMO}=\mathrm{LUMO}-E_{\mathrm{g}} \mathrm{CV} . E_{\mathrm{g}} \mathrm{CV}=$ electrochemical gap.

In order to evaluate the electrical performances of this first generation of oligothiophenerylenimide semiconductors, films were grown and used to build top-contact-bottom gate (TC/BG) field-effect transistors. The films were obtained either by vapor deposition or solution processed onto $\mathrm{Si} / \mathrm{SiO}_{2}$ substrates pretreated with HDMS or OTS. The best degree of crystallinity was found for NDI-1T because of the formation of cofacial $\pi$-conjugated planes aligned to the $\mathrm{Si} / \mathrm{SiO}_{2}$ surface with a calculated tilt angle of $61.2^{\circ}$. In contrast, NDI-3T thin films resulted in an amorphous material, probably due to the lack of planarity caused by a sulfur-oxygen repulsion interaction which forces the disruption of the oligothiophene planarity. Although they are less crystalline than NDI-1T, the presence of sharp Bragg reflections in the X-ray diffractograms of PDI-1T and PDI-3T evidenced the existence of a certain degree of crystallinity in the perylene derivatives.

AFM analyses of the morphology and microstructure for the semiconductors in thin films reveal large planar terraces ascribed to polycrystalline surfaces for NDI-1T (Figure 4) [12]. For the NDI-3T derivative, the catenation of the thiophene backbone is translated in the loss of crystalline grain size, phenomena related to the amorphous profile in XRD. The replacement of the thiophene central ring by phenylene units has a remarkable effect in the microstructure, increasing the grain size. Finally, the introduction of perylenimide units in the semiconductor structure of PDI-1T and PDI-3T leads to well-connected crystallites but with smaller sizes than those observed for NDI-1T. 


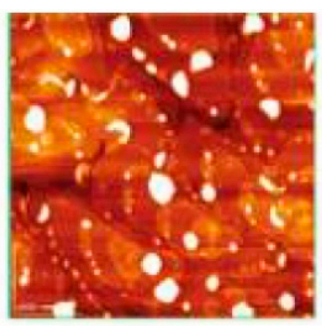

NDI-1T

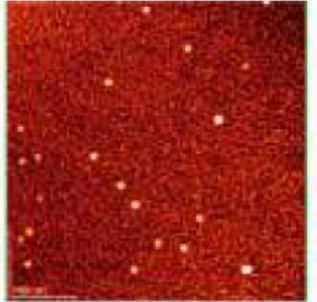

NDI-3T

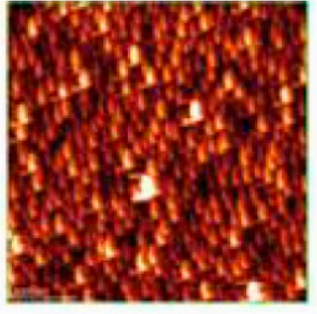

PDI-3T

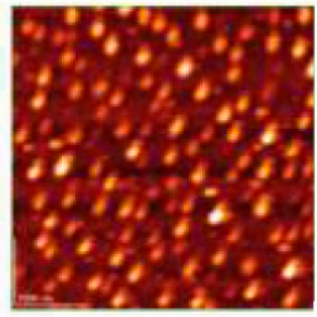

NDI-T-Ph-T

Figure 4. AFM images of vapor-deposited films of NDI-1T $\left(T_{d}=110{ }^{\circ} \mathrm{C}\right.$ on HMDS), NDI-3T ( $T_{d}=25^{\circ} \mathrm{C}$ on HMDS-treated $\mathrm{Si} / \mathrm{SiO}_{2}$ substrates), PDI-3T $\left(T_{d}=110^{\circ} \mathrm{C}\right.$ on HMDS) and NDI-T-Ph-T ( $T_{d}=25^{\circ} \mathrm{C}$ on OTS-treated $\mathrm{Si} / \mathrm{SiO}_{2}$ substrates). Adapted with permission from J. Am. Chem. Soc. 2010, 132, 24, 8440-8452. Copyright@ 2010 American Chemical Society [12].

TC/BG field-effect transistor properties were evaluated in terms of charge carrier mobility $(\mu)$, current on-off ratio $\left(\mathrm{I}_{\mathrm{ON}} / \mathrm{I}_{\mathrm{OFF}}\right)$ and threshold voltage $\left(V_{T}\right)$ (Table 2$)$. The best charge transport characteristics were found for NDI-1T when vapor-deposited films were grown at $110{ }^{\circ} \mathrm{C}$ on $\mathrm{Si} / \mathrm{SiO}_{2}$ surfaces treated with $\mathrm{HMDS}$, with electron mobilities reaching values of $0.35 \mathrm{~cm}^{2} \mathrm{~V}^{-1} \mathrm{~s}^{-1}$. These good results regarding NDI-1T could be explained in terms of high crystallinity and good molecular packing in well-connected grains, as observed in XRD and AFM analyses. The smaller grain sizes and lower crystallinity observed for PDI-1T with a more extended $\pi$-conjugated system accounts for the slightly lower electron mobility of $0.1 \mathrm{~cm}^{2} \mathrm{~V}^{-1} \mathrm{~s}^{-1}$ measured for this system. Regarding naphthalimidebased assemblies, the introduction of the phenylene moiety in NDI-T-Ph-T enhanced the crystallinity and the presence of well-defined and well-connected smaller grains, allowing mobilities of around $0.1 \mathrm{~cm}^{2} \mathrm{~V}^{-1} \mathrm{~s}^{-1}$ for electron transport. Finally, the introduction of catenated thiophene units in both NDI-3T and PDI-3T decreases their performances in the devices by around two orders of magnitude in agreement with the analyses of AFM images and XRD patterns. In contrast with the analogue with one thiophene unit (NDI-1T), the introduction of additional thiophene moieties in NDI-3T produces a twist in the oligothiophene backbone, thus hindering an efficient packing and therefore causing a decrease in the crystallinity of the films. As a result, electron mobilities of only $10^{-4} \mathrm{~cm}^{2} \mathrm{~V}^{-1} \mathrm{~s}^{-1}$ can be measured for these assemblies. In comparison with the naphthalimide containing assembly NDI-3T, films made from the perylenimide-containing analogue, PDI-3T, are more crystalline, having larger grains allowing for better charge carrier mobilities up to $10^{-3} \mathrm{~cm}^{2} \mathrm{~V}^{-1} \mathrm{~s}^{-1}$. Solution-processed OTFT transistors were also fabricated, and their morphologies and crystal structures were evaluated. The results obtained for these semiconductors were in good agreement with the new morphologies obtained for each one and, in the case of PDI-3T, an electrical enhancement was found in comparison with the vapor-deposited thin-film transistors [12].

Table 2. OTFT Electrical data for vapor-deposited films of NDI-1T, NDI-3T, PDI-1T, PDI-3T and NDI-T-Ph-T measured under vacuum on $\mathrm{Si} / \mathrm{SiO}_{2}$ substrates ${ }^{\mathrm{a}}$.

\begin{tabular}{cccccc}
\hline Semiconductor & $\boldsymbol{S}^{\mathbf{b}}$ & $\boldsymbol{T}_{\boldsymbol{d}}\left({ }^{\circ} \mathbf{C}\right)^{\mathbf{c}}$ & $\boldsymbol{\mu}_{\boldsymbol{e}}$ & $\boldsymbol{V}_{\boldsymbol{T}}$ & $\mathrm{I}_{\text {ON }} / \mathbf{I}_{\text {OFF }}$ \\
\hline \multirow{2}{*}{ NDI-1T } & $\mathrm{H}$ & 110 & 0.35 & 28 & $10^{6}$ \\
& $\mathrm{O}$ & 110 & 0.10 & 28 & $10^{5}$ \\
& $\mathrm{~S}$ & 110 & $1.2 \times 10^{-2}$ & 55 & $10^{2}$ \\
\hline \multirow{2}{*}{ NDI-3T } & $\mathrm{H}$ & 110 & $2 \times 10^{-4}$ & 10 & $10^{2}$ \\
& $\mathrm{H}$ & 25 & $3.5 \times 10^{-4}$ & 45 & $10^{3}$ \\
\hline PDI-1T & $\mathrm{H}$ & 110 & 0.15 & 27 & $10^{7}$ \\
\hline
\end{tabular}


Table 2. Cont.

\begin{tabular}{cccccc}
\hline Semiconductor & $\boldsymbol{S}^{\mathbf{b}}$ & $\boldsymbol{T}_{\boldsymbol{d}}\left({ }^{\circ} \mathbf{C}\right)^{\mathbf{c}}$ & $\mu_{\boldsymbol{e}}$ & $\boldsymbol{V}_{\boldsymbol{T}}$ & $\mathrm{I}_{\mathrm{ON}} / \mathrm{I}_{\mathrm{OFF}}$ \\
\hline \multirow{2}{*}{ PDI-3T } & $\mathrm{H}$ & 110 & $1.3 \times 10^{-3}$ & 45 & $10^{4}$ \\
& $\mathrm{O}$ & 110 & $3.5 \times 10^{-2}$ & 45 & $10^{5}$ \\
\hline \multirow{2}{*}{ NDI-T-Ph-T } & $\mathrm{H}$ & 25 & $7.4 \times 10^{-2}$ & 45 & $10^{6}$ \\
& $\mathrm{O}$ & 25 & 0.10 & 58 & $10^{5}$ \\
\hline
\end{tabular}

a Electron carrier mobility $(\mu)$ is given in $\mathrm{cm}^{2} \mathrm{~V}^{-1} \mathrm{~s}^{-1}$ and threshold voltages $\left(V_{T}\right)$ in $\mathrm{V}^{\mathrm{b}}{ }^{\mathrm{b}}$ Device parameters reported are for films grown on untreated $\mathrm{Si} / \mathrm{SiO}_{2}$ substrates $(\mathrm{S})$, hexamethyldisilazane vapor-treated $\mathrm{Si} / \mathrm{SiO}_{2}$ substrates $(\mathrm{H})$ or octadecyltrichlorosilane-treated $\mathrm{Si} / \mathrm{SiO}_{2}$ substrates $(\mathrm{O}) .{ }^{\mathrm{c}}$ Deposition temperature.

It is also worth pointing out that the terthiophene-naphthalimide assemblies (NDI-3T and PDI-3T) are suitable for electropolymerization (Scheme 2) [10,19]. Thus, electropolymerization of PDI-3T generates a functionalized polythiophene with a UV-vis spectrum covering the whole UV-vis region. The polymer has a band gap of $0.9 \mathrm{eV}$ and exhibits ambipolar characteristics, showing high electroactivity in both the $p$ - and the $n$-doping processes [10]. On the other hand, NDI-3T can be also electropolymerized to yield a polymer which can store specific charges of $13 \mathrm{mAh} \mathrm{g}^{-1}$ for polymer oxidation and $38 \mathrm{~mA} \mathrm{~g}^{-1}$ for its reduction, which is interesting for fast charge/discharge batteries [19].
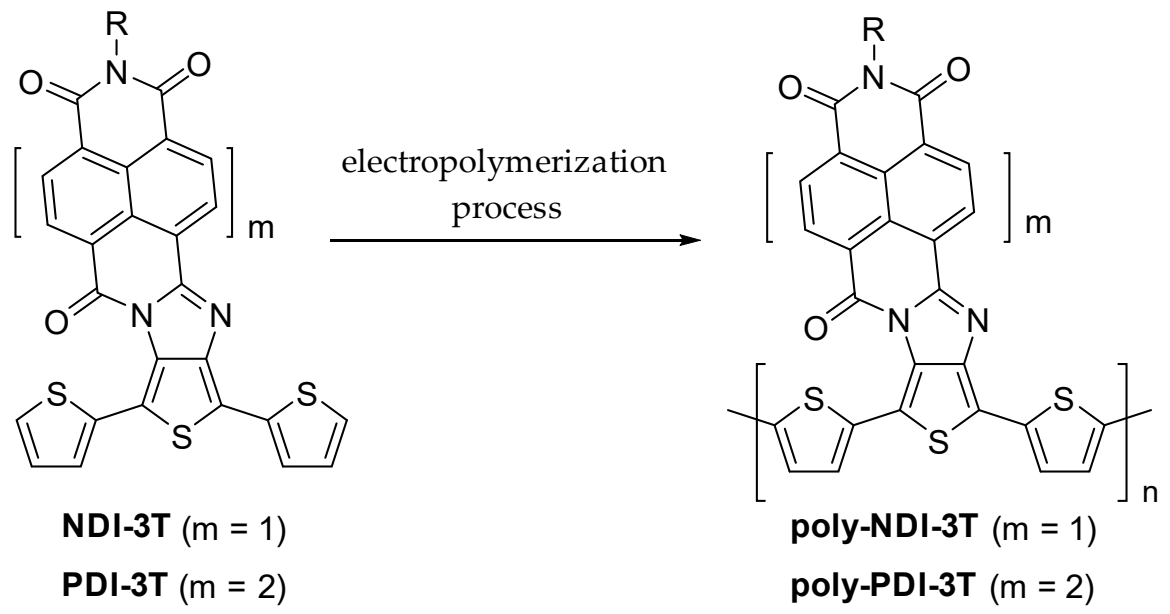

Scheme 2. Synthesis of rylenimide polymers derived from NDI-3T and PDI-3T.

Motivated by the above results [12], a novel family of naphthalimide-oligothiophene assemblies [20] (second-generation NDI-nT) was designed with the aim to avoid the above-described skeletal distortion (Figure 5).
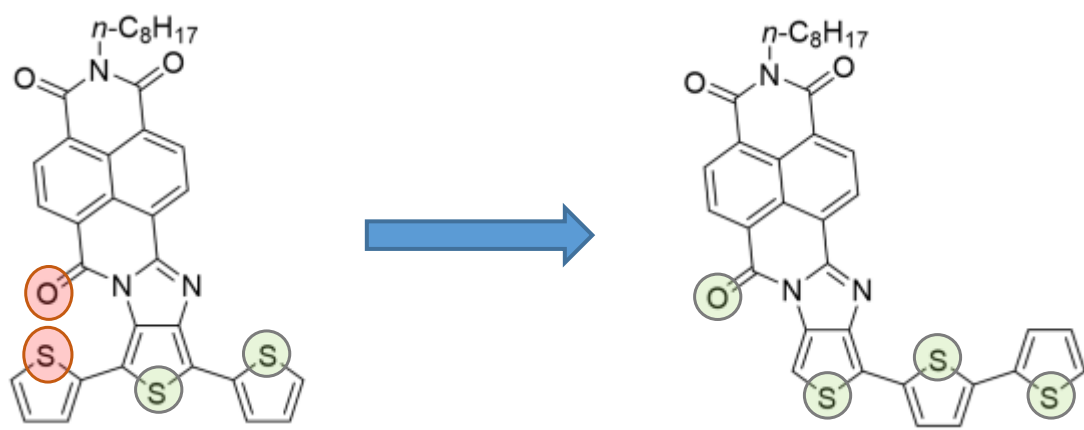

Figure 5. Schematic representation of oxygen-sulfur repulsive interaction (highlighted in red) in the first generation of NDI-oligothiophene derivatives and how it is suppressed in the second generation. 
In order to obtain this second generation of oligothiophene-naphthalimide assemblies, a linear synthetic approach was followed (Scheme 3) instead of the convergent one used previously [10-12]. Thus, a monobrominated thiophene-naphthalimide derivative (NDI1T-Br, Scheme 3) was used as starting material. Stille cross-coupling reactions between NDI-1T-Br and the corresponding monostannane derivatives 4,5 and 6 were carried out in order to obtain the target molecules NDI-2T, NDI-3Tp and NDI-4T in good yields [20]. As depicted in Scheme 3, the steric repulsion between the oxygen and sulfur heteroatoms is absent in these new organic semiconductors, thus preventing the skeletal distortions. Additionally, NDI-5T (Scheme 3) was synthesized for comparison purposes, given that it combines a fragment with the extended $\pi$-conjugated structure of NDI-3Tp and also a fragment with the steric hindrance of NDI-3T. NDI-5T was obtained by a Suzuki crosscoupling reaction between NDI-1T-2Br and the 2,2' - Bithiophene-5-boronic acid pinacol ester (7).

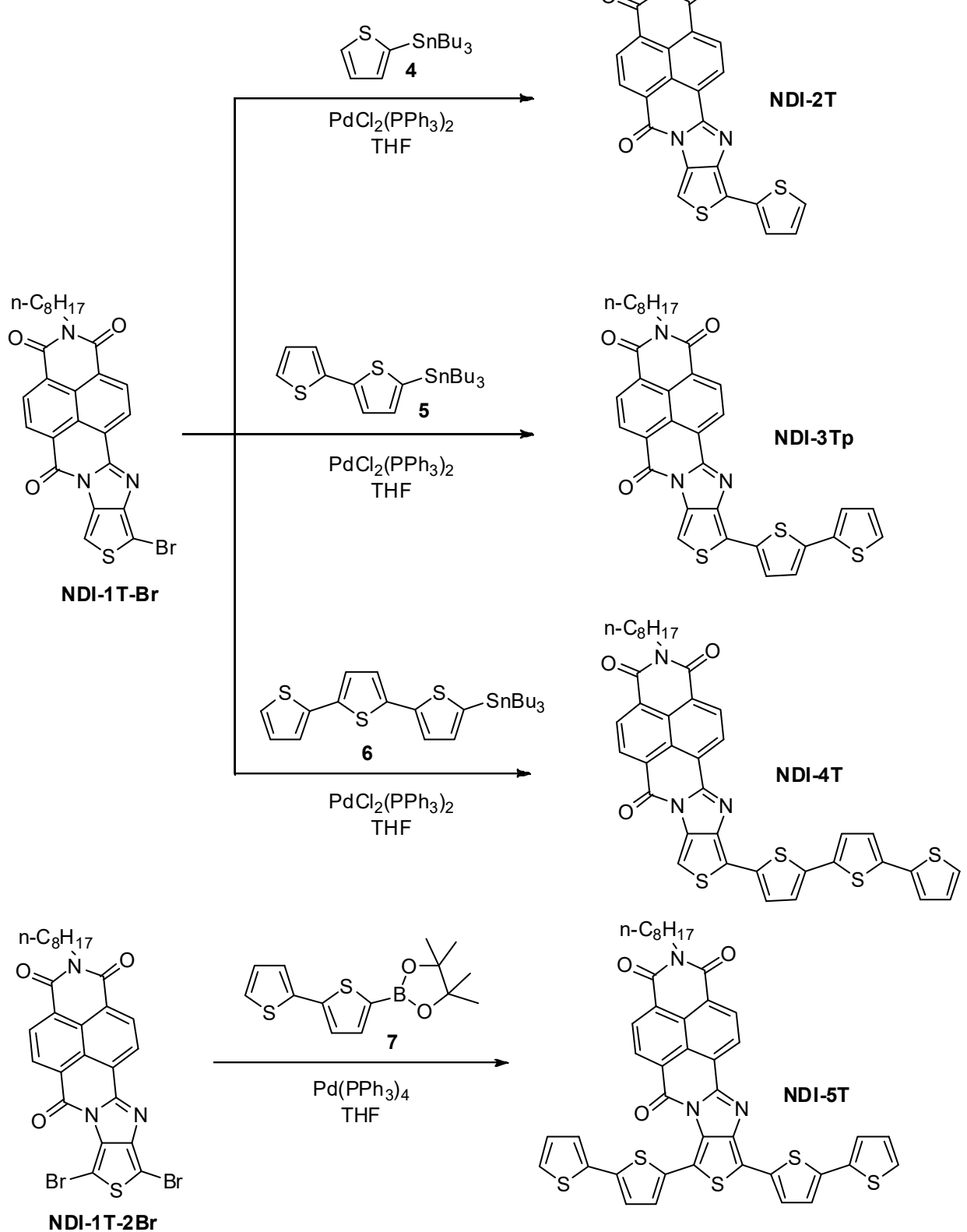

Scheme 3. Synthetic route designed for the NDI-nT materials. 
The UV-vis absorption spectra of this second generation of oligothiophene-naphthalimide assemblies present broad absorption bands above $550 \mathrm{~nm}$, with a significant charge-transfer character. The HOMO/LUMO energy levels for these NDI-nT materials were estimated from the data collected by cyclic voltammetry $[17,18]$. The LUMO energy levels remain practically unaltered independent of the oligothiophene length, while the HOMO energy levels are more destabilized upon increasing the oligothiophene length. This behavior can be rationalized in terms of the LUMO and HOMO molecular topologies obtained by DFT/B3LYP /6-31G ${ }^{* *}$ theoretical calculations. The HOMOs are delocalized over the oligothiophene fragment while the LUMOs are located on the naphthalimide moiety (Figure 6). The HOMO levels of NDI-4T and NDI-5T approach the Fermi level of Au, thus improving the hole injection in electronic devices. Thus, the molecular design used in this strategy allows for the optimization of the $p$-type performance of the semiconductors without sacrificing the electron transport, due to the separation of the frontier molecular orbitals.

It is also worth noting that the band gap of the planar NDI-3Tp (Figure 5, right) is $0.1 \mathrm{eV}$ smaller than that of the NDI-3T analogue with a distorted structure (Figure 5, left). This energy readjustment, in conjunction with other morphological and electronic factors, is probably the origin of the ambipolar behavior shown by NDI-3Tp.
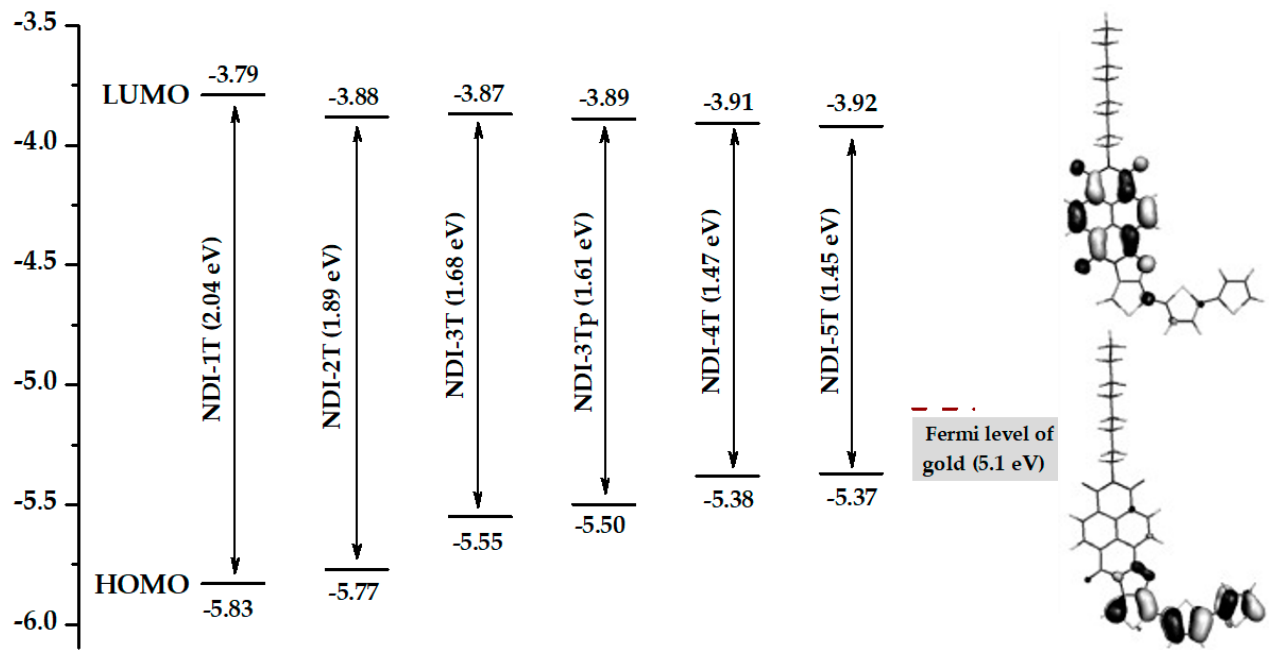

Figure 6. Left: $\mathrm{HOMO} / \mathrm{LUMO}$ energy levels derived from the electrochemical measurements. Energy gaps are shown in brackets. Right: DFT/B3LYP/6-31G** LUMO (top) and HOMO (bottom) orbital molecular topologies of NDI-3Tp. Reproduced with permission from Chem. Eur. J. 2012, 18, 532-543. Copyright@ 2012 WILEY-VCH Verlag GmbH \& Co. KGaA, Weinheim, Germany [20].

Thin films of these NDI-nT semiconducting materials were processed from solutions or by sublimation. X-ray analyses of the thin films show that crystallinity is much more pronounced in those films obtained by sublimation. However, solution processed films are also well textured. For all the laterally monosubstituted semiconductors, NDI-2T, NDI-3Tp and NDI-4T, the films obtained are crystalline, with at least three diffraction peaks. The AFM images of these films show small, well-interconnected crystallite domains. For comparison purposes, thin films of NDI-5T were also analyzed. These films exhibit noncrystalline microstructures which were also confirmed by AFM analysis in which only isolated domains were observed. The lack of crystallinity in the NDI-5T films can be ascribed to the presence of the oxygen-sulfur repulsive interaction, which leads to the loss of planarity in the skeleton structure and, from that, to poorly ordered packing.

The most remarkable characteristics of the FET performances of these semiconductors were investigated in TC/BG OFETs (Table 3). The shortest derivative, NDI-2T, only shows electron mobilities up to $2.6 \times 10^{-2} \mathrm{~cm}^{2} \mathrm{~V}^{-1} \mathrm{~s}^{-1}$. The fact that NDI-2T only exhibit $n$-type mobility values is consistent with the similarity of its HOMO energy level and that of NDI-1T. In contrast, ambipolarity was found for NDI-3Tp and NDI-4T. This ambipolar 
behavior could be related to: (1) the lack of skeletal distortions that allows close $\pi-\pi$ stacking interactions and lower reorganization energies and (2) the presence of longer conjugated oligothiophene moieties with increased $\pi$ conjugation lengths that allows a destabilization of the HOMO energy levels which approach the Fermi level of Au. For NDI3Tp and NDI-4T, the best performances obtained for solution-processed films, annealed at $90{ }^{\circ} \mathrm{C}$, were $2.01 \times 10^{-2} \mathrm{~cm}^{2} \mathrm{~V}^{-1} \mathrm{~s}^{-1}$ and $6.79 \times 10^{-3} \mathrm{~cm}^{2} \mathrm{~V}^{-1} \mathrm{~s}^{-1}$ for electrons and holes, respectively. On the other hand, mobilities of $3.15 \times 10^{-3} \mathrm{~cm}^{2} \mathrm{~V}^{-1} \mathrm{~s}^{-1}$ and $3.15 \times 10^{-3}$ $\mathrm{cm}^{2} \mathrm{~V}^{-1} \mathrm{~s}^{-1}$, for electrons and holes, respectively, were obtained for vapor-deposited films. Interestingly, FETs fabricated using NDI-5T exhibit ambipolar transport behavior with balanced electron and hole transport mobilities of approximately $10^{-5} \mathrm{~cm}^{2} \mathrm{~V}^{-1} \mathrm{~s}^{-1}$. The study demonstrates that the absence of ambipolarity is mainly due to the disruption in the $\pi$ conjugation, while a decrease in the carrier mobility is induced by the skeletal distortions. Furthermore, there is a good correlation between the charge transport properties, the film microstructures and the ordered packing of nondistorted structures.

Table 3. OFET electrical data for vapor-deposited films of NDI-2T, NDI-3Tp, NDI-4T and NDI-5T measured under vacuum on $\mathrm{Si} / \mathrm{SiO}_{2}$ substrates. Average field-effect mobilities are shown ${ }^{\mathrm{a}}$.

\begin{tabular}{ccccccccc}
\hline Semiconductor & $S^{\mathbf{b}}$ & $T_{d}\left({ }^{\circ} \mathbf{C}\right)^{\mathbf{c}}$ & $\mu_{e}$ & $V_{T}$ & $\mathbf{I}_{\mathrm{ON}} / \mathbf{I}_{\mathrm{OFF}}$ & $\mu_{h}$ & $V_{T}$ & $\mathrm{I}_{\mathrm{ON}} / \mathbf{I}_{\mathrm{OFF}}$ \\
\hline \multirow{2}{*}{ NDI-2T } & $\mathrm{H}$ & 110 & $1.41 \times 10^{-2}$ & 31 & $10^{5}$ & - & - \\
& $\mathrm{H}$ & 25 & $2.11 \times 10^{-3}$ & 50 & $10^{5}$ & - & - \\
\hline \multirow{2}{*}{ NDI-3Tp } & $\mathrm{H}$ & 25 & $1.27 \times 10^{-3}$ & 34 & $2 \times 10^{2}$ & - & - & - \\
& $\mathrm{O}$ & 25 & $1.83 \times 10^{-3}$ & 42 & $3 \times 10^{3}$ & $1.67 \times 10^{-4}$ & -62 & $4 \times 10^{2}$ \\
\hline \multirow{2}{*}{ NDI-4T } & $\mathrm{H}$ & 110 & $7.53 \times 10^{-3}$ & 41 & $10^{2}$ & $1.13 \times 10^{-3}$ & -51 & 10 \\
& $\mathrm{H}$ & 25 & $6.37 \times 10^{-5}$ & 28 & $3 \times 10^{2}$ & $4.79 \times 10^{-5}$ & -59 & $10^{2}$ \\
\hline \multirow{2}{*}{ NDI-5T } & $\mathrm{H}$ & 110 & $3.21 \times 10^{-6}$ & 54 & $10^{2}$ & - & - & - \\
\hline
\end{tabular}

a Electron carrier mobility $(\mu)$ is given in $\mathrm{cm}^{2} \mathrm{~V}^{-1} \mathrm{~s}^{-1}$ and threshold voltages $\left(V_{T}\right)$ in $\mathrm{V}$. ${ }^{\mathrm{b}}$ Device parameters reported are for films grown on hexamethyldisilazane vapor-treated $\mathrm{Si} / \mathrm{SiO}_{2}$ substrates $(\mathrm{H})$ or octadecyltrichlorosilane-treated $\mathrm{Si} / \mathrm{SiO}_{2}$ substrates $(\mathrm{O}) .{ }^{\mathrm{c}}$ Deposition temperature.

In line with these observations, we envisaged that the presence of an important dipole moment in these types of donor-acceptor assemblies may tend to pack them in an antiparallel way (Figure 2d), which can prevent suitable HOMO-HOMO overlap between molecules [12,20,21]. As the modulation of dipole moments has been shown to be a key parameter for tuning the electrical performance of organic semiconductors [21-23], with the aim to efficiently tune the molecular packing of organic semiconducting materials, we combined our expertise in the syntheses of oligothiophene-naphthalimide assemblies with new strategies based on tuning dipole moments. Thus, we designed a third generation of bis-naphthalimides 2NDI-nT (Scheme 4) based on a catenated oligothiophene bridge end-capped with NDI-1T structures [24]. We investigated the effect of combining two naphthalimide systems with one oligothiophene moiety in the molecular dipole moments and hence in the organic field-effect transistor performances.

Compounds 2NDI-3Ta, 2NDI-3Tb, 2NDI-4T and 2NDI-5T were obtained by Pdcatalyzed Stille cross-coupling reactions between NDI-1T-1Br and the corresponding bisstannanes 8, 9 and 10. In addition, dimer 2NDI-2T were synthesized by self-dimerization of NDI-1T-1Br with bis(tributyltin) in the presence of $\mathrm{Pd}\left(\mathrm{PPh}_{3}\right)_{4}$ (Scheme 4$)$. 


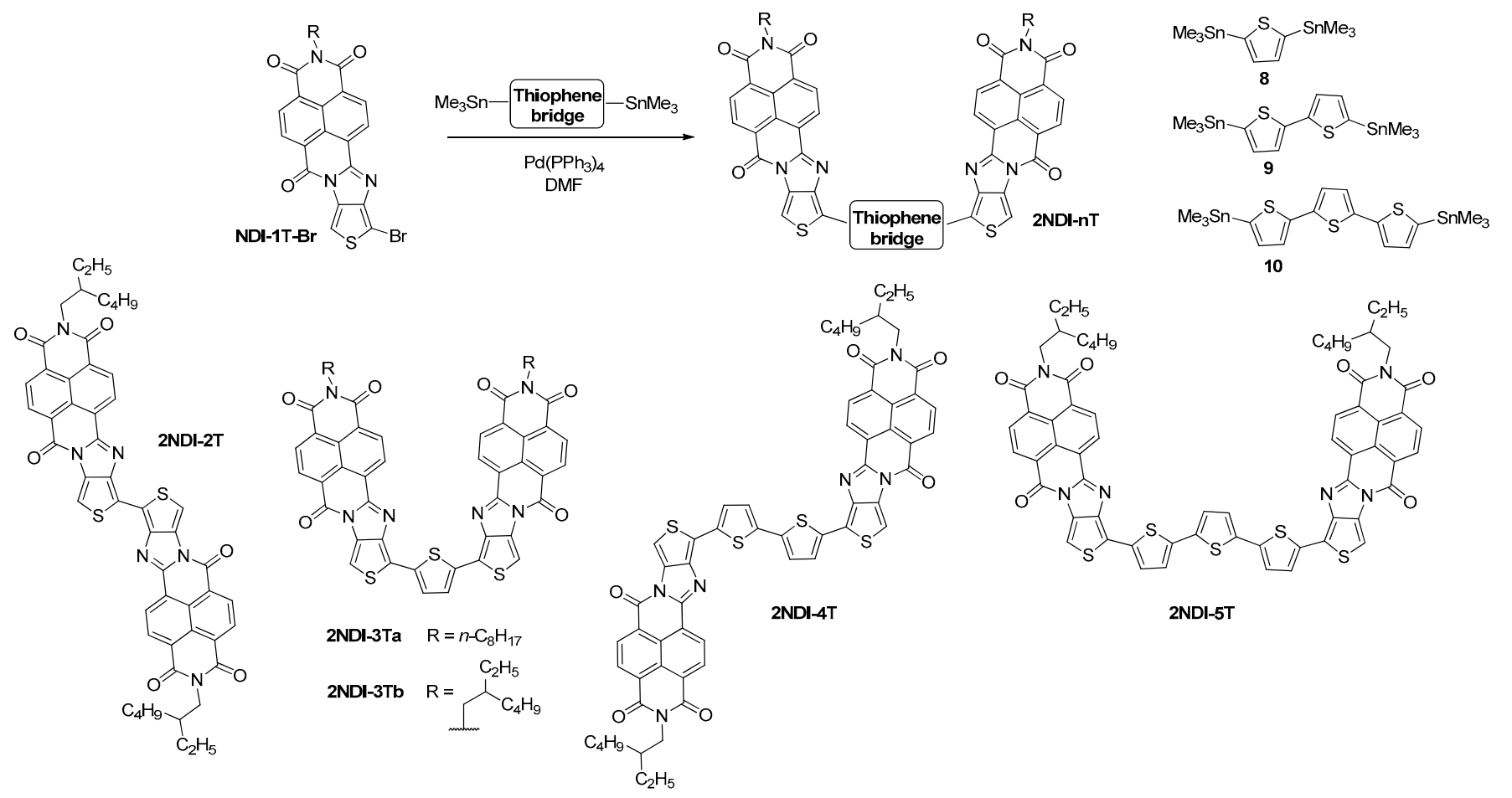

Scheme 4. Schematic representation of the reaction conditions (top) for the syntheses of the third generation of oligothiophene-naphthalimide assemblies (bottom).

All naphthalimide dimers have similar absorption patterns with bathochromic shifts for the assemblies with longer oligothiophene fragments. As in the previous oligothiophenenaphthalimide assemblies, an intramolecular charge transfer band appears as a consequence of the segregated spatial location of the HOMO and LUMO molecular orbitals. Cyclic voltammetry measurements allow for the detection of tetraanion species due to the presence of two naphthalimide moieties. Moreover, in the case of 2NDI-5T, not only was the formation of tetraanion species observed but also the formation of stable radical dications [24]. The estimation of the frontier molecular orbital energy levels from the electrochemical data (Figure 7) shows that there is a destabilization of the HOMO energy level with the catenation. The presence of tetra and pentathiophene in the assemblies approaches the HOMO energy level to the Fermi level of $\mathrm{Au}$, thus favoring the injection of holes and therefore enabling $p$-type transport.

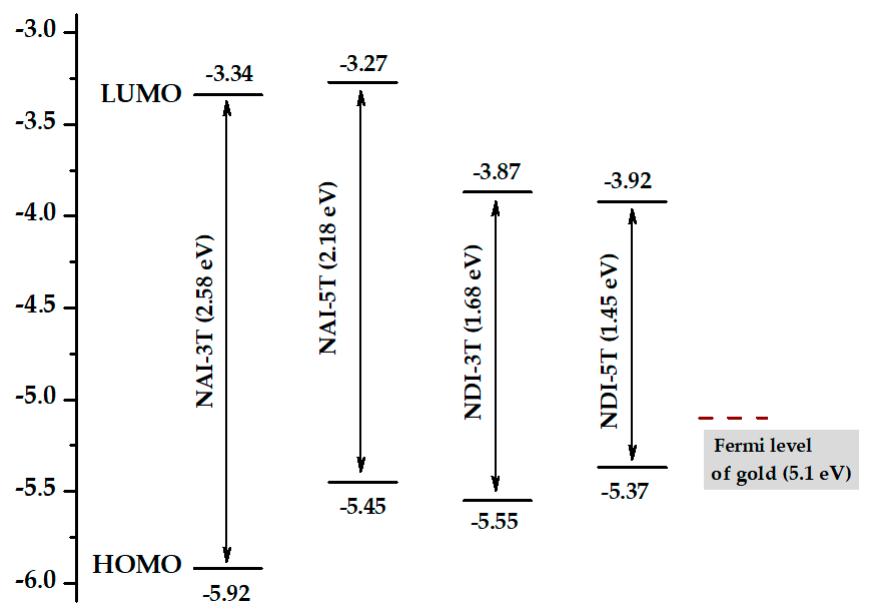

Figure 7. Electrochemically-derived HOMO and LUMO energy levels of 2NDI-nT semiconductors. 
In order to analyze the influence of non-negligible molecular dipole moments in the molecular packing, computational studies were carried out. It was found that for 2NDI-2T and 2NDI-4T, with a pair number of thiophene units, the molecular dipolar moments are rather small, and there are negligible energetic differences between antiparallel and parallel dispositions. On the other hand, the semiconductors with an odd number of thiophene rings have non-negligible molecular dipolar moments and there is a stabilization of parallel stacking versus the antiparallel one.

Thin films of the 2NDI-nT semiconducting materials were fabricated by drop casting from chlorobenzene solutions. Wide-angle X-ray diffraction (WAXRD) analyses show that all these 2NDI-nT films are poorly crystalline, while tapping-mode AFM indicates remarkable differences upon annealing treatment. Thus, more favorable morphologies with rounded well-connected grains can be obtained with higher annealing temperatures.

In Table 4, the most significant characteristics of the FET performances of these semiconductors in TC/BG OFETs are depicted. 2NDI-2T and 2NDI-3T exhibit comparable electron mobilities of $2.5 \times 10^{-4} \mathrm{~cm}^{2} \mathrm{~V}^{-1} \mathrm{~s}^{-1}$ and $3.6 \times 10^{-4} \mathrm{~cm}^{2} \mathrm{~V}^{-1} \mathrm{~s}^{-1}$, respectively. Regarding 2NDI-4T and 2NDI-5T, ambipolar properties were found. Well balanced hole and electron mobilities of $8.4 \times 10^{-3} \mathrm{~cm}^{2} \mathrm{~V}^{-1} \mathrm{~s}^{-1}$ and $1.8 \times 10^{-3} \mathrm{~cm}^{2} \mathrm{~V}^{-1} \mathrm{~s}^{-1}$ were found for 2NDI-5T. Interestingly, 2NDI-4T transports electrons more efficiently, while hole transport is more favored for 2NDI-5T. These results are in good agreement with the estimated HOMO and LUMO energy levels. Nevertheless, the changes in the molecular dipole moments could not be directly correlated with the charge carrier mobility values.

Table 4. OFET electrical data for vapor-deposited films of 2NDI-nT semiconductors measured under vacuum $^{\mathrm{a}}$.

\begin{tabular}{|c|c|c|c|c|c|c|c|}
\hline Semicond. & $\begin{array}{c}\text { Annealing } \\
\text { Temperature }^{b}\end{array}$ & $\mu_{e}$ & $V_{T}$ & $\mathrm{I}_{\mathrm{ON}} / \mathrm{I}_{\mathrm{OFF}}$ & $\mu_{h}$ & $V_{T}$ & $\mathbf{I}_{\mathrm{ON}} / \mathrm{I}_{\mathrm{OFF}}$ \\
\hline \multirow{3}{*}{ 2NDI-2T } & At cast & $2.1 \times 10^{-5}$ & 2.6 & $8 \times 10^{3}$ & - & - & - \\
\hline & 180 & $2.5 \times 10^{-4}$ & 5.3 & $2 \times 10^{4}$ & - & - & - \\
\hline & 250 & $1.9 \times 10^{-4}$ & 1.6 & $2 \times 10^{4}$ & - & - & - \\
\hline \multirow{3}{*}{ 2NDI-3Ta } & At cast & $2.4 \times 10^{-6}$ & 18.1 & $10^{2}$ & - & - & - \\
\hline & 180 & $2.3 \times 10^{-6}$ & 22.3 & 70 & - & - & - \\
\hline & 250 & $7.6 \times 10^{-6}$ & 23 & $2 \times 10^{2}$ & - & - & - \\
\hline \multirow{3}{*}{ 2NDI-3Tb } & At cast & $3.3 \times 10^{-4}$ & 20 & $7 \times 10^{4}$ & - & - & - \\
\hline & 180 & $3.6 \times 10^{-4}$ & 13 & $7 \times 10^{4}$ & - & - & - \\
\hline & 250 & $3.0 \times 10^{-4}$ & 20.2 & $3 \times 10^{5}$ & - & - & - \\
\hline \multirow{3}{*}{ 2NDI-4T } & At cast & $2.0 \times 10^{-2}$ & 13.4 & $5 \times 10^{2}$ & $2.6 \times 10^{-4}$ & -43 & $8 \times 10^{2}$ \\
\hline & 180 & $5.8 \times 10^{-3}$ & 8.25 & $10^{4}$ & $2.7 \times 10^{-4}$ & -27.5 & 40 \\
\hline & 250 & $2.5 \times 10^{-4}$ & 5.35 & $2 \times 10^{4}$ & $1.1 \times 10^{-5}$ & -17 & $5 \times 10^{2}$ \\
\hline \multirow{3}{*}{ 2NDI-5T } & At cast & $1.5 \times 10^{-4}$ & 15 & $8 \times 10^{2}$ & $6.7 \times 10^{-6}$ & -36 & $7 \times 10^{2}$ \\
\hline & 180 & $1.3 \times 10^{-3}$ & 16.6 & $3 \times 10^{2}$ & $4.3 \times 10^{-4}$ & -28.9 & $8 \times 10^{2}$ \\
\hline & 250 & $1.8 \times 10^{-3}$ & 17.3 & 70 & $8.4 \times 10^{-3}$ & -27.1 & 10 \\
\hline
\end{tabular}

${ }^{a}$ Electron carrier mobility $(\mu)$ is given in $\mathrm{cm}^{2} \mathrm{~V}^{-1} \mathrm{~s}^{-1}$ and threshold voltages $\left(V_{T}\right)$ in $\mathrm{V} .{ }^{\mathrm{b}}$ Temperature is given in ${ }^{\circ} \mathrm{C}$.

\section{Inverted Amidine-Based Oligothiophene-Naphthalimide Semiconductors}

The lessons learned from the first three series of oligothiophene-naphthalimide assemblies allowed us to conclude that ambipolar semiconductors with these kinds of systems can be obtained by (i) connecting the donor and acceptor moieties through conjugated rigid linkers, (ii) avoiding steric hindrance and skeletal distortions and (iii) extending the effective $\pi$-conjugated length of the oligothiophene moiety.

In the search for new low-bandgap materials in which facile hole and electron injection are possible from a single type of electrode, we designed a fourth generation of oligothiophene-naphthalimide assemblies, NAI derivatives (Figure 8), that involves the inversion of the amidine linkage connections between the oligothiophenes and the naphthalimide in order to prevent steric interactions (Figure 8) [25]. 


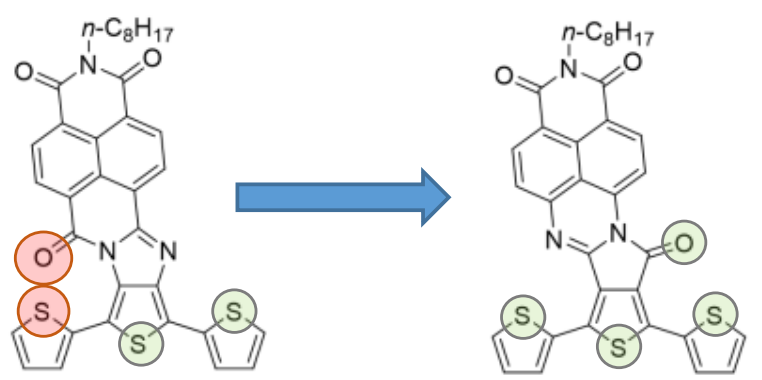

Figure 8. Illustration of the oxygen-sulfur interaction in NDI-3T and NAI-3T derivatives.

As it is depicted in Scheme 5, for the syntheses of the NAI derivatives, naphthalimide moieties endowed with amino functionalities (11) and a thiophene derivative endowed with anhydride functionality (12) were used as starting materials.

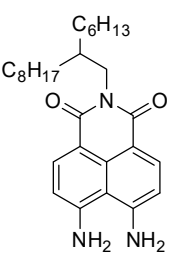

11
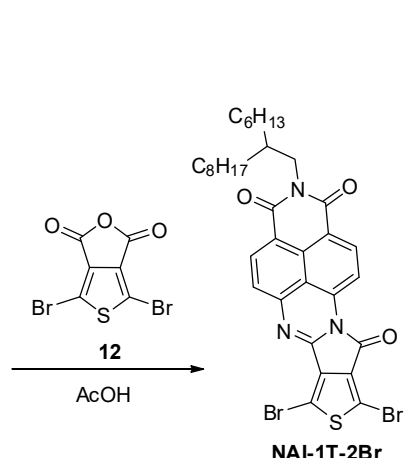

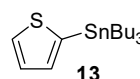

13 $\mathrm{Pd}(\mathrm{dba})_{2} / \mathrm{P}(\mathrm{o}-\mathrm{tol})_{3}$
Toluene/DMF

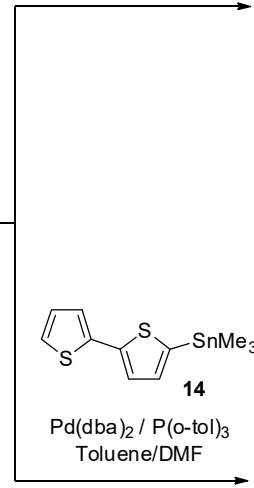

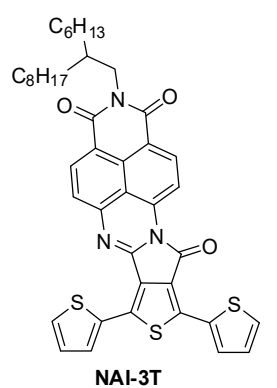

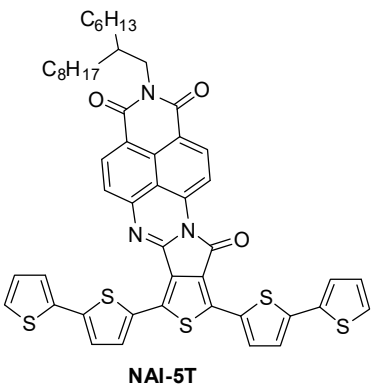

Scheme 5. Syntheses of the fourth generation of oligothiophene-naphthalimide assemblies.

Through a condensation reaction between monomers 11 and 12, the dibrominated NAI-1T-2Br derivative can be obtained. Further reaction of NAI-1T-2Br by Stille crosscoupling reactions with the corresponding stannylated thiophene $\mathbf{1 3}$ or bithiophene $\mathbf{1 4}$ yield the target molecules NAI-3T and NAI-5T. In terms of the molecular structure, the inversion in the orientation of the carbonyl group in the imidazole unit provides more planar semiconductors, in which the O...S repulsive interaction is deliberately avoided in contrast with that observed in the first generation of oligothiophene-naphthalimide assemblies with noninverted amidine linkers $[12,20]$.

The LUMO and HOMO molecular topologies of NAI-3T and NAI-5T could be also obtained by DFT/B3LYP /6-31G * $^{* *}$ theoretical calculations (Figure 9). It is worth pointing out that, in contrast with the parent NDI-3T and NDI-5T derivatives, in the derivatives with the inverted amidine linkers, the HOMO is not localized solely on the oligothiophene fragment, and it is spread over the molecular skeleton. Additionally, in contrast with the NDI derivatives, for NAI-3T and NAI-5T, the LUMO orbital is delocalized not only on the naphthalimide moiety but also in the oligothiophene fragment. 


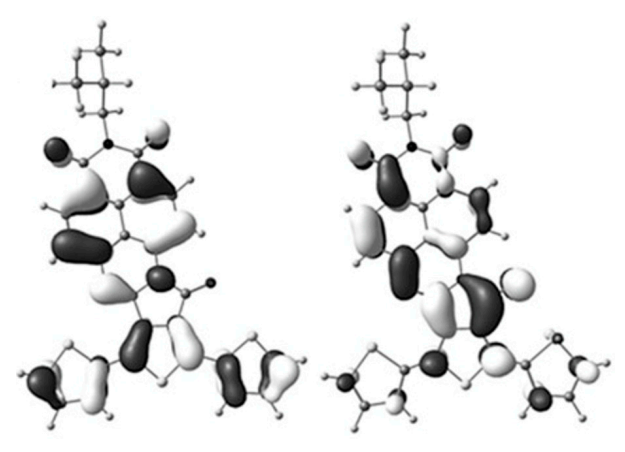

(a)

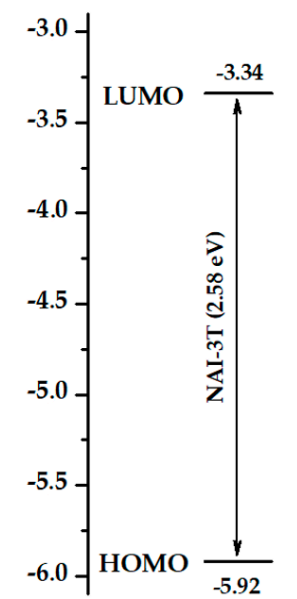

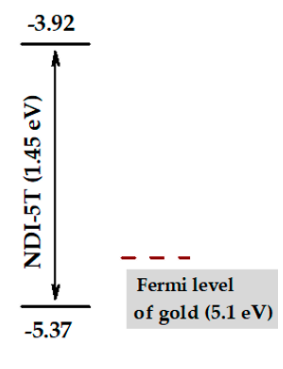

(b)

Figure 9. (a) LUMO (left) and HOMO (right) molecular topologies obtained by DFT7B3LYP76-31G** for NAI-3T. Adapted with permission from Chem. Eur. J. 2016, 22, 13643-13652. Copyright@ 2016 WILEY-VCH Verlag GmbH \& Co. KGaA, Weinheim, Germany [25]. (b) HOMO/LUMO energy levels for oligothiophene-naphthalimide assemblies based on NDI and NAI.

Therefore, there is not a clear separation between the location of the HOMO and LUMO orbitals in NAI derivatives and, given this, the HOMO-LUMO transition does not involve a charge-transfer character in contrast with that observed for the parent NDI analogues. Furthermore, the $\lambda_{\max }$ in the UV-vis absorption spectrum for the NAI derivatives displays hyperchromic and hypsochromic shifts in comparison with that of the parent NDI analogues.

Cyclic voltammetry measurements for both NAI-3T and NAI-5T present oxidation and reduction processes. From these values, the HOMO and LUMO energy levels could be estimated (Figure 9).

The LUMO energy levels estimated for the NAI derivatives are significantly destabilized in comparison with those of the NDI analogues, while the HOMO energy levels of both NAI-3T and NAI-5T are more stabilized than those corresponding to their NDI counterparts $[12,20,24]$. The molecular dipole moment is enhanced by about 4.5 times for the NAI derivatives in comparison with that of the NDI analogues, and smaller hole reorganization energies were obtained for the inverted amidines, which is a good indicator of facile charge accommodation.

Finally, in order to evaluate the electrical properties of these new semiconductors, TC/BG field-effect transistors were fabricated by vapor deposition. The NAI-based films were characterized by WAXRD and AFM. Referring to the crystal packaging, the introduction of inverted amidine in the assemblies leads to more crystalline films in comparison with the amorphous films obtained for the NDI analogues. The high molecular dipole moments of the NAI derivatives reveals a significant tendency for packing with antiparallel orientations [20,24]. The AFM images and the X-ray diffractograms of films prepared from NAI-3T and NAI-5T show evidence of a high dependence of the morphology and crystallinity of the films with the temperature of deposition. Films obtained by sublimation onto OTS-treated surfaces at $110{ }^{\circ} \mathrm{C}$ show larger rod-like crystallites in the case of NAI-3T, while NAI-5T shows regular and small interconnected grains, both in good agreement with the diffraction patterns.

The OFET performance obtained for the vapor-deposited films is summed up in Table 5. In this semiconductor series, $p$-type and ambipolar transport properties were found for NAI-5T and NAI-3T, respectively. In the shorter member of the series, the electron mobilities are similar to that of the NDI analogues, around $1.9 \times 10^{-4} \mathrm{~cm}^{2} \mathrm{~V}^{-1}$ $\mathrm{s}^{-1}$, with interesting balanced values for hole mobilities of $2.0 \times 10^{-4} \mathrm{~cm}^{2} \mathrm{~V}^{-1} \mathrm{~s}^{-1}$ at 25 and $110^{\circ} \mathrm{C}$. Although the outputs for NAI-5T show clear ambipolarity, only hole-charge 
transport values of $5.75 \times 10^{-5} \mathrm{~cm}^{2} \mathrm{~V}^{-1} \mathrm{~s}^{-1}$ were ultimately measured. The better hole mobility values obtained for NAI-5T in comparison with the parent NDI-5T could be related to the HOMO delocalization over the whole molecule, which allows better orbital overlapping between molecules.

Table 5. OFET electrical data for vapor-deposited films of NAI-3T and NAI-5T measured under vacuum on $\mathrm{Si} / \mathrm{SiO}_{2}$ substrates. Average field-effect mobilities are shown ${ }^{\mathrm{a}}$.

\begin{tabular}{ccccccccc}
\hline Semiconductor & $S^{\mathbf{b}}$ & $T_{\boldsymbol{d}}\left({ }^{\circ} \mathbf{C}\right)^{\mathbf{c}}$ & $\mu_{e}$ & $\boldsymbol{V}_{\mathrm{T}}$ & $\mathrm{I}_{\mathrm{ON}} / \mathrm{I}_{\mathrm{OFF}}$ & $\mu_{h}$ & $\boldsymbol{V}_{\boldsymbol{T}}$ & $\mathrm{I}_{\mathrm{ON}} / \mathrm{I}_{\mathrm{OFF}}$ \\
\hline \multirow{2}{*}{ NAI-3T } & $\mathrm{O}$ & 110 & $1.15 \times 10^{-4}$ & 42 & 10 & $2 \times 10^{-4}$ & -40 & $5 \times 10^{2}$ \\
& $\mathrm{O}$ & 25 & $1.95 \times 10^{-3}$ & 39 & 20 & $2 \times 10^{-3}$ & -46 & $3 \times 10^{2}$ \\
\hline NAI-5T & $\mathrm{H}$ & - & - & - & - & $6 \times 10^{-5}$ & -41 & $10^{2}$
\end{tabular}

a Electron carrier mobility $(\mu)$ is given in $\mathrm{cm}^{2} \mathrm{~V}^{-1} \mathrm{~s}^{-1}$ and threshold voltages $\left(V_{T}\right)$ in $\mathrm{V} .{ }^{\mathrm{b}}$ Device parameters reported are for films grown on hexamethyldisilazane vapor-treated $\mathrm{Si} / \mathrm{SiO}_{2}$ substrates $(\mathrm{H})$ or octadecyltrichlorosilane-treated $\mathrm{Si} / \mathrm{SiO}_{2}$ substrates $(\mathrm{O}) .{ }^{\mathrm{c}}$ Deposition temperature.

The implementation of these D-A semiconductors into polymeric structures [26] were carried out as depicted in Figure 10. Due to the avoided O $\cdots S$ repulsive interaction in NAI derivatives, we envisioned more planar structures in the polymers based on this structural moiety. For comparison purposes, we also synthesized NDI-based polymeric analogues where the $\mathrm{O} \cdots \mathrm{S}$ repulsive interaction is still present.

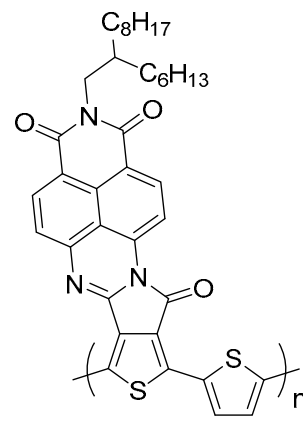

poly(NAI-2T)

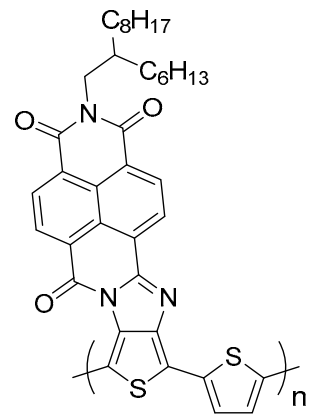

$\operatorname{poly}(\mathrm{NDI}-2 \mathrm{~T})$

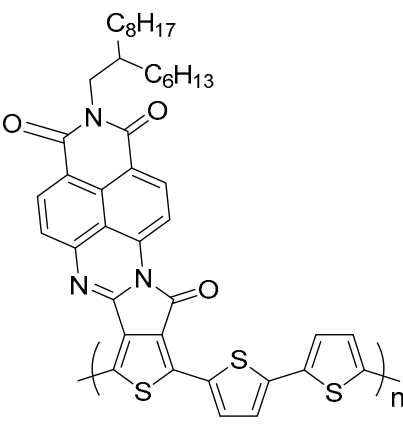

poly(NAI-3T)

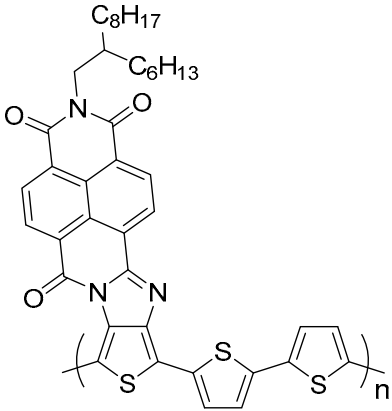

$\operatorname{poly}(\mathrm{NDI}-3 \mathrm{~T})$

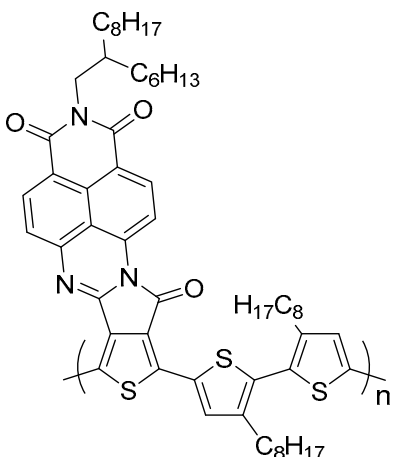

poly(NAI-8C-3T)

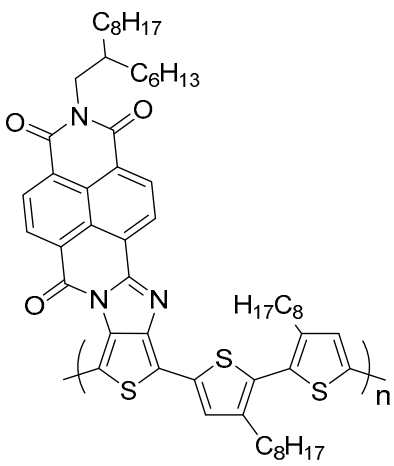

poly(NDI-8C-3T)

Figure 10. Different organic polymers based on NAI-nT and NDI-nT semiconductors.

The polymers could be obtained by Stille cross-coupling reactions between NAI-1T2Br (Scheme 5) or NDI-1T-2Br (Scheme 3) and the corresponding thiophene or bithiophene bisstanannes. Due to the low solubility of poly(NDI-3T) and poly(NAI-3T) derivatives, alkyl chains were introduced in the bithiophene comonomer to obtain more processable materials, designated as poly(NDI-8C-3T) and poly(NAI-8C-3T) (Figure 10). 
The DFT theoretical calculations (Figure 11) carried out for poly(NAI-nT) polymers show the lack of significant $\mathrm{O} \cdots \mathrm{S}$ repulsive interaction, thus yielding to the planarization of the polythiophene backbone in NAI-based polymers. In contrast, in the poly(NDInT) polymers, $\mathrm{O} \cdots \mathrm{S}$ repulsive interactions are present, disrupting to a certain extent the effective conjugation of the polythiophene backbone.
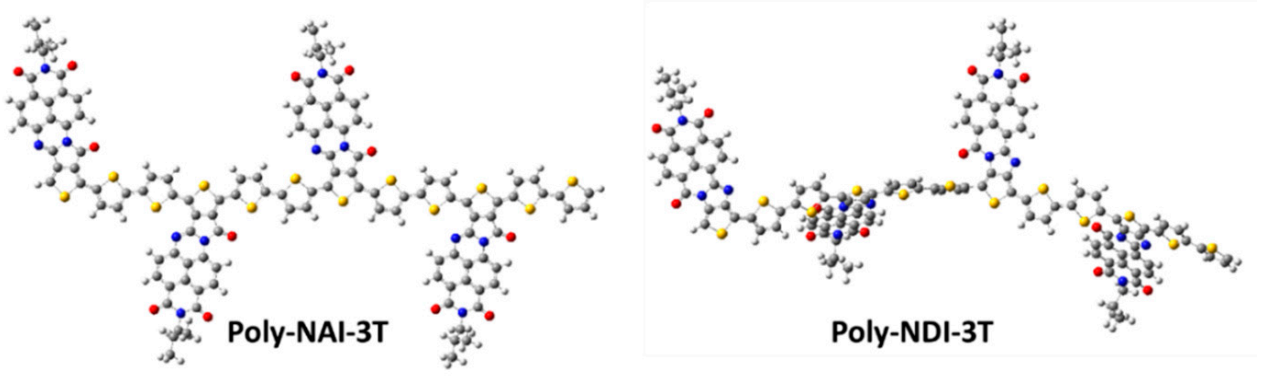

Figure 11. Geometry optimization obtained by DFT/B3LYP/6-31G** theoretical calculations for Poly(NAI-3T) and Poly(NDI-3T).

In the absorption spectra, both poly(NAI-nT) and poly(NDI-nT) derivatives show bathochromic shifts in comparison with the respective molecular analogues due to the extension of the $\pi$-conjugated polymer backbones. Absorption in the whole UV-vis spectrum can be observed, extending even to the near infrared region. Cyclic voltammetry measurements of polymers of the NDI series show well-defined redox processes which contrast with the broad waves exhibited by the analogues of the NAI series. This may be due to the rotational torsion of the NDI-based polythiophene backbones due to the $\mathrm{O} \cdots \mathrm{S}$ repulsive interaction, leading to reduced $\mathrm{p}$ orbital overlap. This interruption of the conjugation produces a sequence of oligothiophene-naphthalimide assemblies connected through nonefficiently conjugated regions. This structural motif accounts for the defined redox processes which are characteristic of molecular systems. In contrast, polymers of the NAI series do not have these $\mathrm{O} \cdots \mathrm{S}$ repulsive interactions, and therefore the polymer backbone can be considered as a conjugated polymer chain with more extended delocalization, thus presenting broad waves which are characteristics of highly delocalized conjugated polymers. The relative nonprocessable nature of the polymers has prevented, until now, the achievement of significant charge carrier mobilities in OFET devices.

\section{Pyrazine-Based Oligothiophene-Rylenimide Semiconductors}

As it was previously shown, avoiding steric hindrance and skeletal distortions in naphthalimide-oligothiophene assemblies plays a crucial role in the search for ambipolarity. For that reason, we designed a fifth generation of naphthalimide-oligothiophene assemblies in which flat and rigid pyrazine units are used as conjugated linkers (Figure 12).<smiles></smiles>

Figure 12. General structure of the fifth generation of oligothiophene-naphthalimide assemblies. 
Pyrazine-based materials can be obtained by condensation reactions between naphthalene (NID) or peryleneimides (PID) endowed with 1,2-diketone functionalities and the corresponding diaminothiophene derivatives (Scheme 6) [27,28].<smiles>[R]c1oc([R])c(N)c1N</smiles><smiles></smiles>

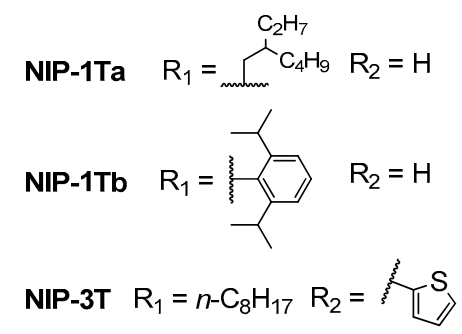<smiles>[R1]n1c(=O)c2ccc3c4ccc5c(=O)c(=O)c6ccc(c7ccc(c1=O)c2c37)c4c56</smiles><smiles>[R]c1sc(C)c(N)c1N</smiles>

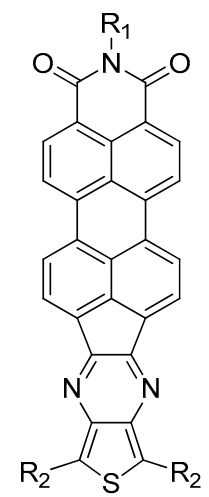

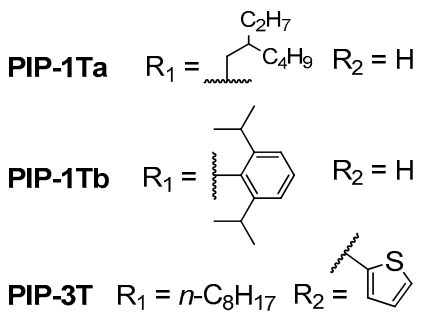

Scheme 6. Syntheses of oligothiophene-naphthalimide (NIP) and perylenimide (PIP) assemblies with pyrazine linkers.

The molecular geometry of the pyrazine-based derivatives was optimized by DFT /B3LYP / 6-31G** theoretical calculations, showing completely planar structures (Figure 13) [28,29]. In NIP-3T and PIP-3T, the absence of steric repulsions and the presence of stabilizing S $\cdots \mathrm{N}$ interactions favored the coplanarity of the oligothiophene-ryleneimide assemblies. DFT theoretical calculations show that the LUMO is mainly localized over the rylenimide core, as in the amidine-based analogues $[12,20,24,25]$. On the other hand, the $\mathrm{HOMO}$ distribution depends on the electron-donor fragment catenation, being located over the conjugated thienopyrazine fragment for the NIP-1T and PIP-1T semiconductor and spread over the whole oligothiophene fragment in the extended oligothiophene derivatives NIP-3T and PIP-3T. As previously mentioned, internal reorganization energies $(\lambda)$ are related to the capability to stabilize injected charges in the system. The obtained values regarding electron and hole charges revealed that $\lambda_{\mathrm{e}}$ for NIP derivatives were comparable to those obtained for NDI. Lower values were obtained in terms of intramolecular reorganization energies for hole charge carriers for NIP derivatives $(0.43 \mathrm{eV}$ of NDI-3T vs. $0.25 \mathrm{eV}$ of NIP-3T), being especially remarkable in the case of NIP-1Ta, where $\lambda_{h}$ was $0.16 \mathrm{eV}$ as a result of the completely planar structure [29]. The extension of the $\pi$-conjugated system provides even lower internal reorganization energies for both electron and hole transport, highlighting the case of PIP-1Ta with $\lambda_{\mathrm{e}}$ and $\lambda_{\mathrm{h}}$ of 0.25 and $0.11 \mathrm{eV}$, respectively [28]. 

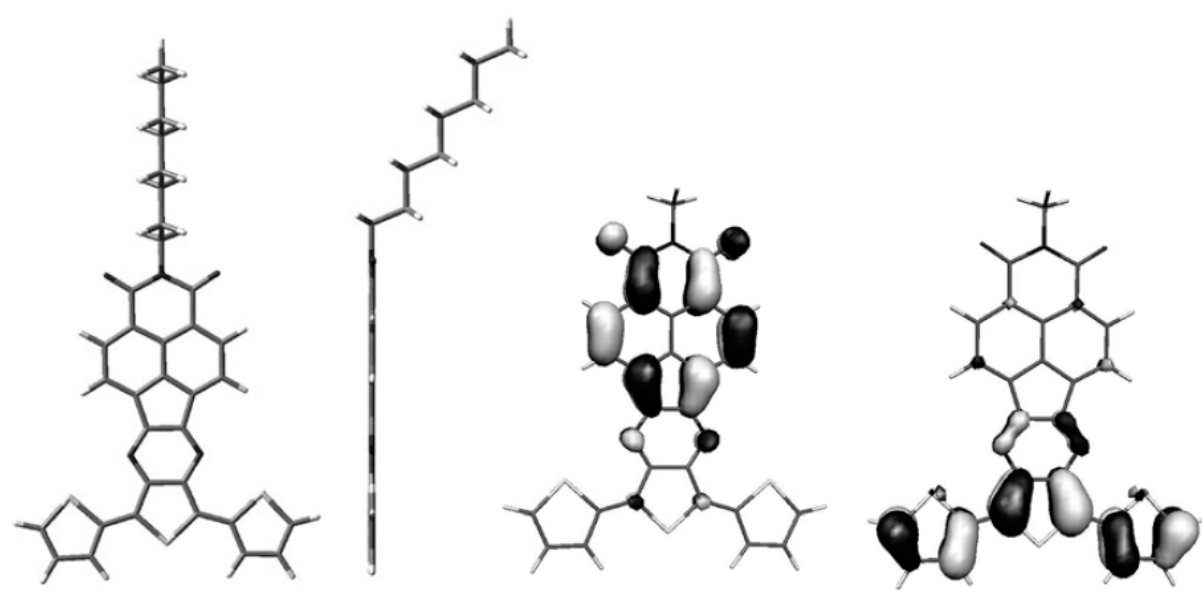

NIP-3T

Figure 13. DFT / / B3LYP /6-31G** geometry optimization (left), LUMO (center) and HOMO (right) of NIP-3T. Adapted with permission from Chem. Eur. J. 2013, 19, 12458-12467. Copyright@ 2013 WILEY-VCH Verlag GmbH \& Co. KGaA, Weinheim, Germany [29].

The absorption spectra of NIP-3T show two absorption bands, in which the lowest energy absorption is centered at $570 \mathrm{~nm}$ and can be ascribed to an ICT excitation, similar to that found for most of the above generations of oligothiophene-naphthalimide assemblies $[12,20,24,25,29]$. This one-electron HOMO-LUMO excitation was also observed in PIP-3T as a broad absorption band centered at $668 \mathrm{~nm}$ with an onset value above $900 \mathrm{~nm}$. Moreover, the characteristic absorption pattern for the perylenimide core is found at 507 and $545 \mathrm{~nm}$, corresponding, respectively, to $0-0$ and $0-1$ vibronic bands of the S0-S1 transition, and at $476 \mathrm{~nm}$ for the S0-S2 transition. The observed differences in the UV-vis spectra were related to the different $\pi$-extended structures of the rylenimide-fused thienopyrazine derivatives. Furthermore, the introduction of different alkyl or phenylene chains at the imide group has no significant effect on their optical properties [28].

In order to study the electrochemical properties of these planar pyrazine-based assemblies, cyclic voltammetry experiments were carried out, and from the obtained electrochemical values, frontier molecular orbital energies could be estimated. As we previously described, oligothiophene chain catenation had an important effect on the HOMO energy, showing destabilized values as the oligothiophene grows, whereas LUMO energies were barely altered $[20,24]$. The lowest unoccupied molecular orbital energy levels depend on the extension of the rylenimide units. Thus, the estimated LUMO values for NIP-1T and PIP-1T are $-3.40 \mathrm{eV}$ and $-3.49 \mathrm{eV}$, respectively. Regarding the oligothiophene counterpart, an increment in the number of the thiophenes has a negligible effect in the LUMO level, while this chemical modification clearly affects the HOMO energy levels, and therefore the optical band gap. The HOMO energy level estimated for PIP-1T ( $-5.34 \mathrm{eV})$ is significantly stabilized in comparison to that estimated for PIP-3T $(-4.75 \mathrm{eV})$. The destabilization of the HOMO observed for PIP-3T is in good agreement with the extension of the thiophene backbone and with the absorption spectra. Therefore, in these pyrazine-based semiconductors, tuning the HOMO-LUMO bandgap can be achieved by two approximations: (i) by extending the rylenimide core without modifying the donor moiety and (ii) through the increment in the length of the oligothiophene backbone. Comparison between the HOMO and LUMO energy levels of the NIP and PIP semiconductors and those of the NDI and PDI analogues reveals that the replacement of the imidazole linker by a rigid pyrazine heterocycle is reflected in more destabilized HOMO and LUMO orbitals, as well as in the enlargement of the band gap (Table 6) [28,29]. 
Table 6. Electrochemical potentials versus SCE in $\mathrm{CH}_{2} \mathrm{Cl}_{2}$ (referenced to $\mathrm{Fc} / \mathrm{Fc}^{+}$couple) of NIP-1T, NIP-3Ta, PIP-1T and PIP-3T and frontier molecular orbital energies estimated from CV data.

\begin{tabular}{ccccccccc}
\hline Semiconductor & $E_{\text {red1 }}$ & $E_{\text {red2 }}$ & $E_{\text {ox1 }}$ & $E_{\text {ox2 }}$ & $E_{\mathbf{g}}{ }^{\text {CV }}$ & $E_{\mathbf{g}}{ }^{\text {opt }}$ & LUMO $^{\mathbf{b}}$ & HOMO \\
\hline NIP-1Ta & -0.86 & -1.30 & $1.78^{\mathbf{a}}$ & - & 2.64 & 2.51 & -3.58 & $-6.22^{\mathrm{c}}$ \\
\hline NIP-1Tb & -0.95 & -1.33 & - & - & - & 2.51 & -3.49 & $-6.00^{\mathrm{d}}$ \\
\hline NIP-3Ta & -0.83 & -0.93 & $0.99^{\mathrm{a}}$ & $1.75^{\mathrm{a}}$ & 1.68 & - & -3.61 & $-5.43^{\mathrm{c}}$ \\
\hline PIP-1Ta & -0.72 & -0.99 & - & - & - & 2.01 & -3.72 & $-5.73^{\mathrm{d}}$ \\
\hline PIP-1Tb & -1.04 & -1.28 & - & - & - & 1.94 & -3.40 & $-5.34^{\mathrm{d}}$ \\
\hline PIP-3T & -1.11 & -1.41 & - & - & - & 1.43 & -3.33 & $-4.76^{\mathrm{d}}$ \\
\hline
\end{tabular}

$\overline{{ }^{a}}$ Irreversible. ${ }^{\mathrm{b}}$ LUMO energy level estimated vs. vacuum level from $E_{\mathrm{LUMO}}=-4.44 \mathrm{eV}-\mathrm{e} E_{\mathrm{red} 1} \cdot{ }^{\mathrm{c}}$ Estimated from $\mathrm{HOMO}=\mathrm{LUMO}-E_{\mathrm{g}} \mathrm{CV} . E_{\mathrm{g}}{ }^{\mathrm{CV}}=$ electrochemical gap. ${ }^{\mathrm{d}}$ Estimated from HOMO $=\mathrm{LUMO}-E_{\mathrm{g}}{ }^{\text {opt }} . E_{\mathrm{g}}{ }^{\text {opt }}=$ optical gap.

As a common evaluation of the electrical properties for these pyrazine-based oligothiophene-rylenimide derivatives, the fabrication of TC/BG thin-film organic fieldeffect transistors was accomplished. Moreover, the thin films obtained for all the semiconductors measured were also studied by AFM and XRD analyses. AFM analysis of NIP-1T, NIP-3T, PIP-1T and PIP-3T (Figure 14) reveals a strong dependence of the deposition temperature on the microstructure domains, doubling the grain size in the case of NIP-1T when the temperature is increased from $25^{\circ} \mathrm{C}$. Interestingly, for PIP-3T, large rod-like grain crystallites were observed, as was the case for the naphthalene analogue NIP-3T. In addition, the effect of the imide substitution was evaluated by comparing the AFM images of PIP-1Ta and PIP-1Tb, which revealed that the presence of the phenyl units enhances the formation of well-rounded grains as a result of a better connectivity and order. The thin-film microstructures of NIP-3T were compared with those of the amidine-based analogues, NDI-3T and NDI-3Tp, because of their structural similarities. By using wide-angle X-ray diffraction (WAXRD) techniques, we observed that films of NDI-3Tp and NIP-3T are relatively crystalline, while those of NDI-3T were essentially amorphous. Nevertheless, the crystallinity of NDI-3Tp is the highest, showing Bragg reflections up to the sixth order.
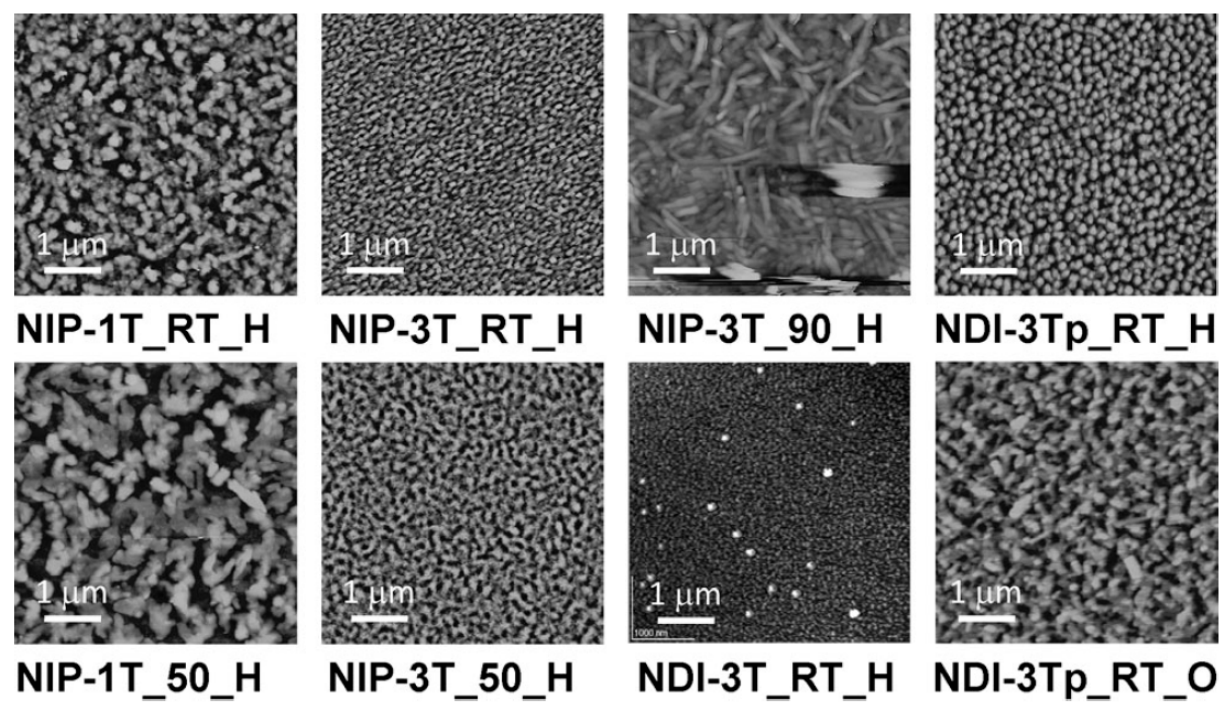

NDI-3T_RT_H NDI-3Tp_RT_O

Figure 14. AFM images of vapor-deposited thin films of NDI-3T, NDI-3Tp and NIP-3T. Reproduced with permission from Chem. Eur. J. 2013, 19, 12458-12467. Copyright@ 2013 WILEY-VCH Verlag $\mathrm{GmbH} \&$ Co. KGaA, Weinheim, Germany [29]. 
Concerning the charge transport ability of these thienopyrazine derivatives, all of them showed $n$-type mobilities, with the best electron mobility value of $2.87 \times 10^{-4} \mathrm{~cm}^{2}$ $\mathrm{V}^{-1} \mathrm{~s}^{-1}$ for NIP-1T when it was deposited at $50{ }^{\circ} \mathrm{C}$ on HMDS-treated dielectric (Table 7). NIP-3T is the only thienopyrazine derivative that showed ambipolar behavior, with good balanced values for both hole and electron charge carriers. This effect is related to the high planar structure and low internal reorganization energies, enabling both electron and hole transport processes. The same behavior could be expected for PIP-3T, but only discrete n-type mobility was observed $\left(1.2 \times 10^{-4} \mathrm{~cm}^{2} \mathrm{~V}^{-1} \mathrm{~s}^{-1}\right)$. This result could be explained in terms of the formation of antiparallel $\pi-\pi$ stackings among the molecules when they were vapor deposited over the dielectric, restricting the formation of the $\mathrm{D}$ and $\mathrm{A}$ domains necessary for the hole and electron transporting. This aggregation behavior is in good agreement with the predicted computational study for dimeric species [28].

Table 7. OFET electrical data for vapor-deposited films of NIP-1Ta, NIP-3Ta, PIP-1T and PIP-3T measured under vacuum on vapor-treated $\mathrm{Si} / \mathrm{SiO}_{2}$ substrates. Average field-effect mobilities are shown ${ }^{\mathrm{a}}$.

\begin{tabular}{|c|c|c|c|c|c|c|c|c|}
\hline Semiconductor & $S^{\mathbf{b}}$ & $T_{d}\left({ }^{\circ} \mathrm{C}\right)^{\mathrm{c}}$ & $\mu_{e}$ & $V_{\mathrm{T}}$ & $\mathrm{I}_{\mathrm{ON}} / \mathrm{I}_{\mathrm{OFF}}$ & $\mu_{h}$ & $V_{T}$ & $\mathrm{I}_{\mathrm{ON}} / \mathrm{I}_{\mathrm{OFF}}$ \\
\hline \multirow{2}{*}{ NIP-1Ta } & $\mathrm{H}$ & 50 & $2.9 \times 10^{-4}$ & 47 & $3 \times 10^{4}$ & - & - & - \\
\hline & $\mathrm{H}$ & 25 & $1.3 \times 10^{-5}$ & 49 & $3 \times 10^{3}$ & - & - & - \\
\hline \multirow{2}{*}{ NIP-3Ta } & $\mathrm{H}$ & 90 & $4.3 \times 10^{-5}$ & 47 & $4 \times 10^{4}$ & $4.10 \times 10^{-5}$ & -47 & $3 \times 10^{3}$ \\
\hline & $\mathrm{H}$ & 25 & $1.0 \times 10^{-4}$ & 71 & $7 \times 10^{2}$ & $1.38 \times 10^{-5}$ & -65 & $5 \times 10^{2}$ \\
\hline \multirow{3}{*}{ PIP-1Ta } & $\mathrm{H}$ & 150 & $1.1 \times 10^{-4}$ & 33 & - & - & - & - \\
\hline & $\mathrm{O}$ & 150 & $1.5 \times 10^{-4}$ & 36 & - & - & - & - \\
\hline & $\mathrm{H}$ & 110 & $5.8 \times 10^{-5}$ & 31 & - & - & - & - \\
\hline PIP-1Tb & $\mathrm{O}$ & 110 & $1.2 \times 10^{-4}$ & 25 & - & - & - & - \\
\hline PIP-3T ${ }^{d}$ & $\mathrm{O}$ & 130 & $1.2 \times 10^{-4}$ & 8 & - & - & - & - \\
\hline
\end{tabular}

The above studies presented for the different generations of oligothiophene-naphthalimide assemblies show that there is an important effect of the crystalline structure as well as of the thin-film microdomains on the charge transport properties. In this regard, the tuning of the supramolecular packing has proven to be critical to obtain proper spatial arrangements of the donor-acceptor assemblies where heterojunctions are clearly segregated into donor and acceptor channels, promoting efficient charge transport. This strategy was firstly addresses by the introduction of hydrophilic or hydrophobic termini side chains in order to promote different self-assembled domains in the previously reported NIP-3T core [29,30]. Thus, we envisaged that the formation of highly ordered aggregates is more probable for NIP-3 $\mathbf{T}_{\text {amphi }}$ bearing hydrophobic chains at the imide nitrogen and ethylene glycol hydrophilic chains on the oligothiophene moiety than in the case of the semiconductor

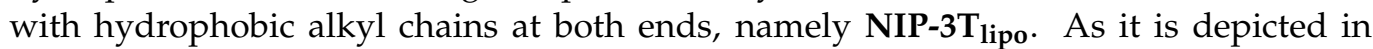
Scheme 7, the syntheses of these new derivatives based on oligothiophene-naphthalimide assemblies were carried out following the convergent method previously described for these materials $[28,29]$. 

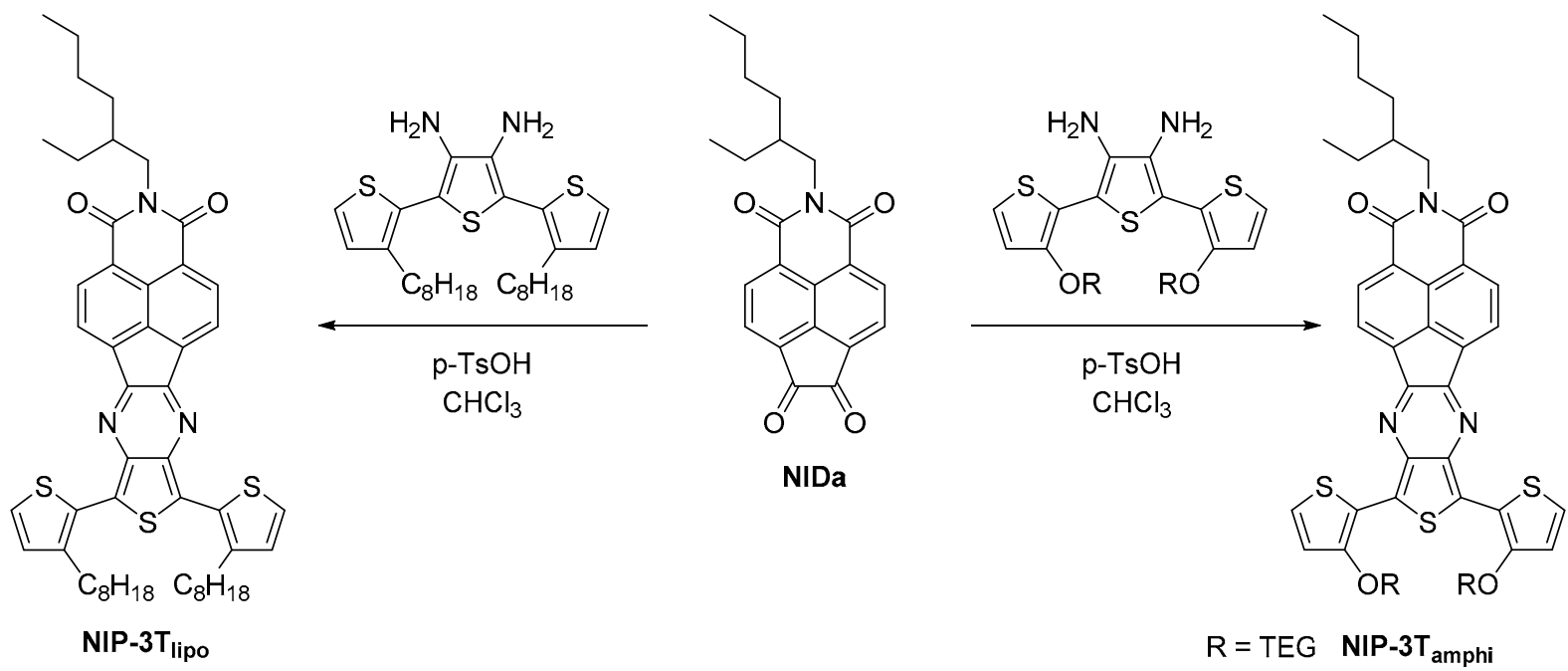

Scheme 7. Synthetic procedure for the semiconducting materials NIP-3T lipo $_{\text {and NIP-3T }}$ amphi

In order to study the (opto)electronic properties of these novel materials, cyclic voltammetry and UV-vis absorption measurements were carried out, and all values are summarized in Table 8. The HOMO/LUMO energy levels estimated by the electrochemical measurements are practically identical in both assemblies, with similar values of the LUMO and negligible differences in the HOMO due to the presence of different terminiside chains on the oligothiophene moiety. Concerning the absorption properties, both materials have similar absorption profiles in comparison with the unfunctionalized NIP-

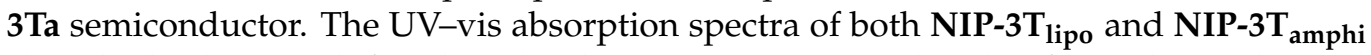
show bathochromic shifts when the absorption is measured in thin films, thus indicating considerable aggregation behavior for both materials.

Table 8. Summed optical and electrochemical values obtained for the absorption and cyclic voltammetry measurements of NIP-3T amphi $_{\text {and NIP-3T }}$ lipo.

\begin{tabular}{|c|c|c|c|c|c|c|c|c|c|c|}
\hline Compound & $\lambda_{\max }(\mathrm{nm})$ & $\lambda_{\text {ons }}(\mathrm{nm})$ & $E_{\mathrm{g}}^{\text {opt }}$ & $E_{\text {red1 }}{ }^{a}$ & $E_{\text {red2 }}{ }^{a}$ & $E_{\text {ox1 }}{ }^{\mathrm{a}, \mathrm{b}}$ & $E_{\text {ox2 }}{ }^{\mathrm{a}, \mathrm{b}}$ & LUMO $^{c}$ & HOMO $^{d}$ & $E_{\mathrm{g}} \mathrm{CV} \mathrm{e}$ \\
\hline $\mathrm{NIP} 3 \mathrm{~T}_{\mathrm{amphi}}$ & 346.520 & 639 & 1.94 & -1.32 & -1.70 & 0.55 & - & -3.78 & -5.65 & 1.87 \\
\hline $\mathrm{NIP} 3 \mathrm{~T}_{\text {lipo }}$ & 345.526 & 659 & 1.88 & -1.33 & -1.72 & 0.49 & 0.68 & -3.77 & -5.59 & 1.82 \\
\hline
\end{tabular}

${ }^{a}$ Electrochemical potentials versus SCE in $\mathrm{CH}_{2} \mathrm{Cl}_{2}$ (referenced to $\mathrm{Fc} / \mathrm{Fc}^{+}$couple). ${ }^{\mathrm{b}}$ Anodic potentials. ${ }^{\mathrm{c}}$ LUMO energy level estimated from $E_{\mathrm{LUMO}}=-\left(E_{\mathrm{red} 1}+5.1\right)(\mathrm{eV}) .{ }^{\mathrm{d}} \mathrm{HOMO}$ level estimated from $E_{\mathrm{HOMO}}=-\left(E_{\mathrm{ox} 1}+5.1\right)(\mathrm{eV}) .{ }^{\mathrm{e}} E_{\mathrm{g}}{ }^{\mathrm{CV}}=E_{\mathrm{LUMO}}-E_{\mathrm{HOMO}}$.

Interestingly, when the absorption measurements are carried out in $\mathrm{THF} / \mathrm{H}_{2} \mathrm{O}$ solvent mixtures, some differences were observed. When the THF $/ \mathrm{H}_{2} \mathrm{O}$ ratio varies from $100 / 0$

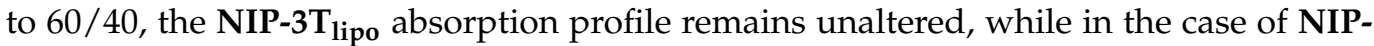
$3 \mathbf{T}_{\mathbf{a m p h i}}$, the lower energy absorption is redshifted and the spectra become similar to that obtained for the thin film, which suggests the formation of supramolecular aggregates. To further study the different tendency toward aggregation of these assemblies, hexane solutions were prepared for both NIP-3T amphi $_{\text {and NIP-3T }}$ lipo. Upon heating at $60{ }^{\circ} \mathrm{C}$ and subsequent cooling at $25^{\circ} \mathrm{C}$, a clear solution was obtained for NIP-3T by performing the same thermal treatment, a suspension resulted for NIP-3T $\mathbf{a m p h i}$. After drying the suspension, analysis by scanning electron microscopy (SEM) showed the formation of microfibers longer than $20 \mu \mathrm{m}$ with a high aspect ratio (Figure 15). By using good solvent/bad solvent mixtures it was also possible to grow micrometer-sized rods

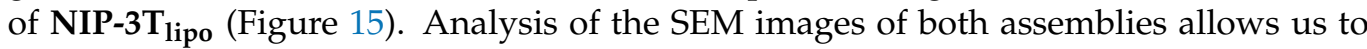
conclude that the microstructures formed are significantly different due to the different substitution pattern. 
To rationalize the different nanostructure growth for these materials, DFT calculations at CAM-B3LYP level were used to predict the more stable dimeric aggregates for each semiconductor. This functional predicts that parallel disposition is the most stable of the two possible conformations for NIP-3 $\mathbf{T}_{\mathbf{a m p h i}}$, as it confirms the interaction energy values depicted in Figure 16, due to the presence of attractive interactions between the ethylene glycol end moieties. On the contrary, the presence of aliphatic alkyl chains in the terthiophene moiety has less impact in the conformational stabilization, being only $1 \mathrm{kcal} / \mathrm{mol}$ more stabilized the antiparallel model, as it was previously described for other oligothiophene-naphthalimide assemblies [12].

Finally, the charge carrier mobilities of NIP-3T amphi $_{\text {and NIP-3T }}$ lipo were analyzed using time of flight (TOF) techniques [31,32]. Remarkably, it was found that both materials show ambipolar characteristics as it was previously described for the NIP-3T analogue. Interestingly, it can be observed that the introduction of self-assembled directors leads to higher electron and holes mobilities for NIP-3T amphi $\left(4.3 \times 10^{-6} \mathrm{~cm}^{2} \mathrm{~V}^{-1} \mathrm{~s}^{-1}\right.$ and $2.1 \times$ $\left.10^{-6} \mathrm{~cm}^{2} \mathrm{~V}^{-1} \mathrm{~s}^{-1}\right)$ in comparison with those measured for NIP-3T lipo $\left(1.3 \times 10^{-7} \mathrm{~cm}^{2} \mathrm{~V}^{-1}\right.$ $\mathrm{s}^{-1}$ and $\left.6.9 \times 10^{-8} \mathrm{~cm}^{2} \mathrm{~V}^{-1} \mathrm{~s}^{-1}\right)$.

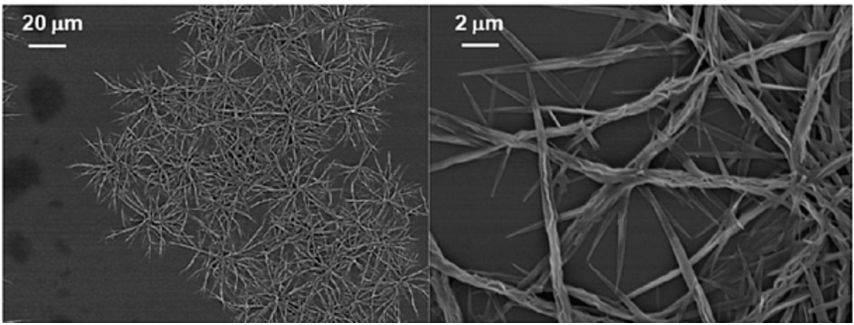

(a)

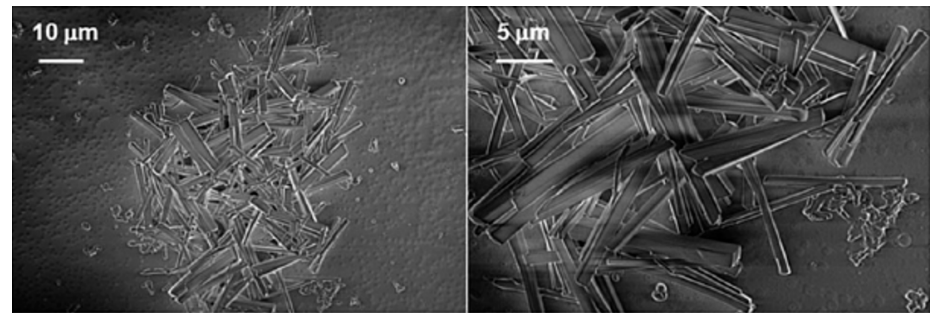

(b)

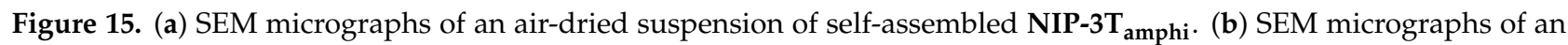

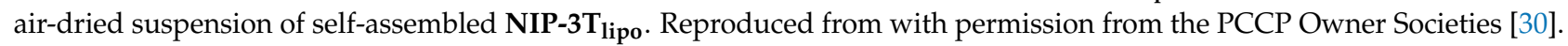
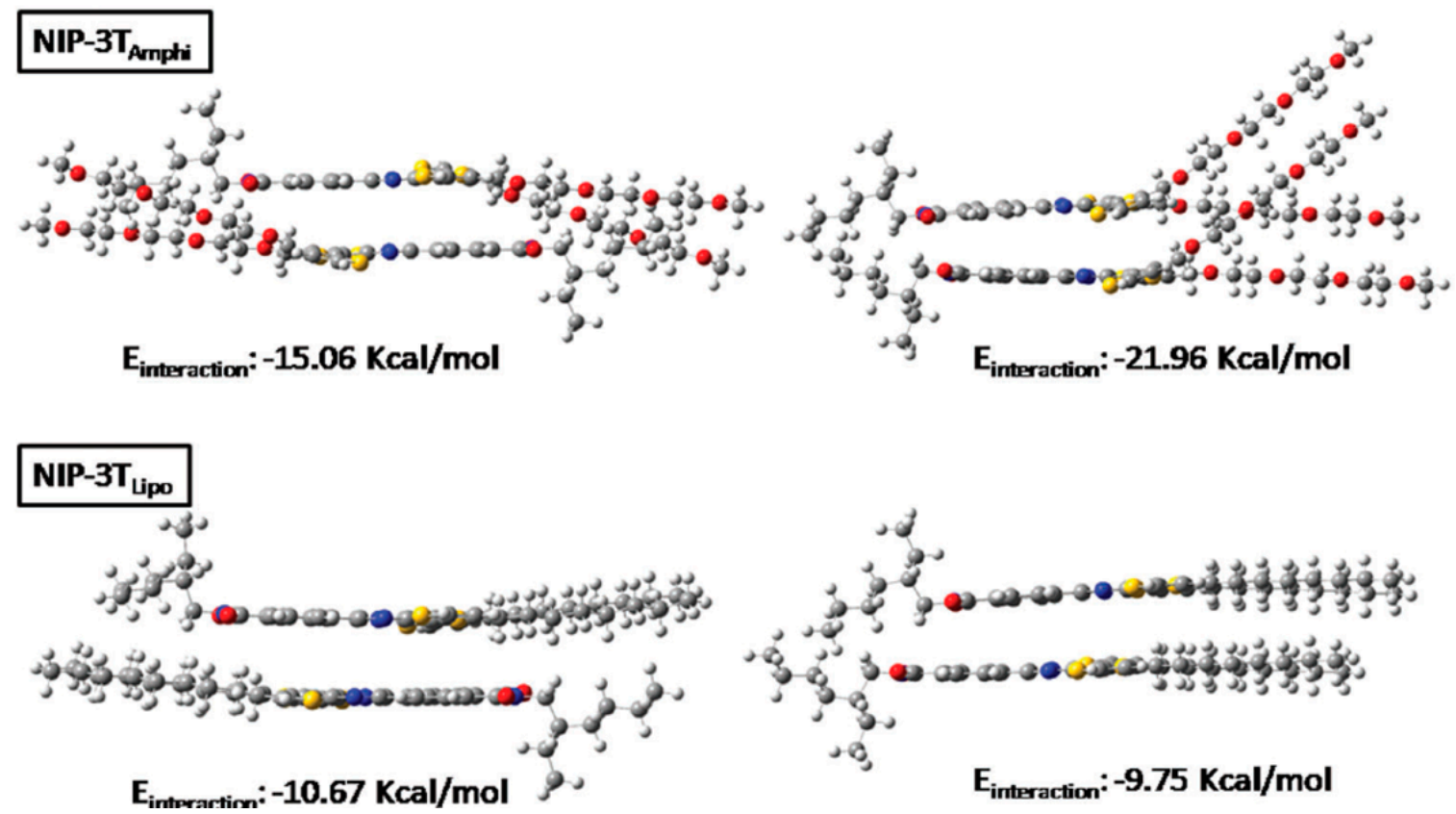

Figure 16. CAM-B3LYP/6-31G** estimated dimers (left: antiparallel configuration (AP) and right: parallel configuration (P)) for NIP-3T amphi $_{\text {and NIP-3T }}$ lipo. Reproduced from with permission from the PCCP Owner Societies [30]. 
To further explore the possibility of tuning the performance of this type of material in (opto)electronic devices, we designed a family of oligothiophene-naphthalimide assemblies based on pyrazine linkers in which end-capped units were introduced in order to promote good packing in the molecule while the HOMO/LUMO energy levels are still suitable for semiconducting applications [33].

These new thienopyrazine-based semiconductors are endowed with different substituents in order to promote molecular ordering, better processability and tuning of the frontier molecular orbitals. These oligothiophene-naphthalimide assemblies can be divided into different groups depending on the nature of the end-capped units introduced: (i) substituents that promote intermolecular interactions and (ii) different electron acceptors in order to modulate the HOMO/LUMO energy levels. Thus, there are three end-capped derivatives functionalized with pyrene, triisopropilsilyl and diphenylamine with a donoracceptor-donor (D-A-D) structure (Scheme 8). On the other hand, the introduction of electron acceptor substituents via Knoevenagel-like reactions led to obtaining A2-D-A1-DA2 semiconductors endowed with rhodanine and dicyanovinylene end groups (Scheme 9).
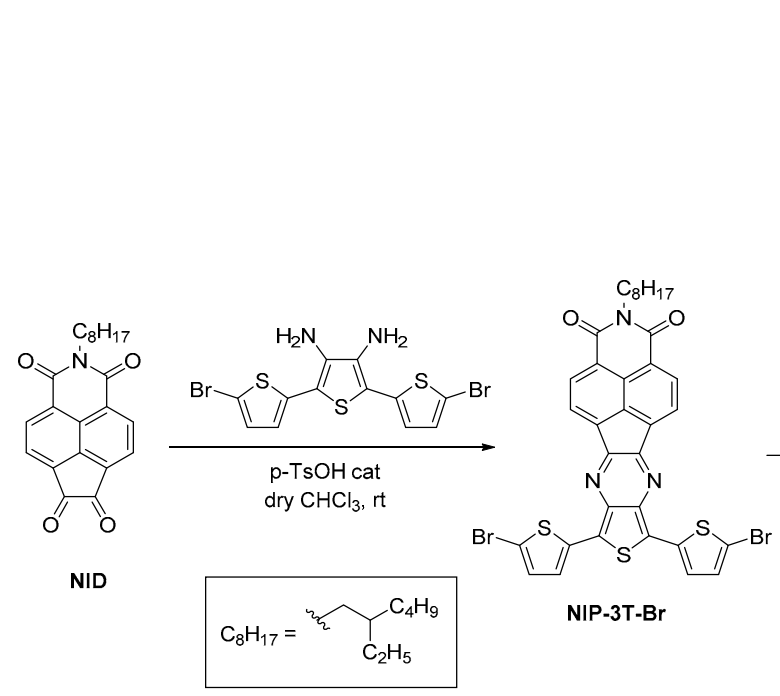
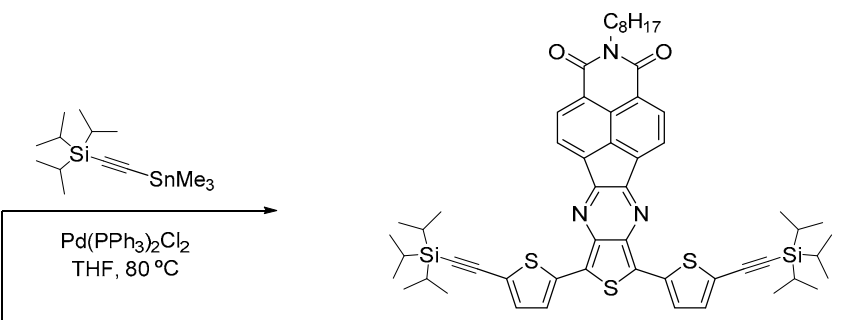

NIP-3T-TIPS
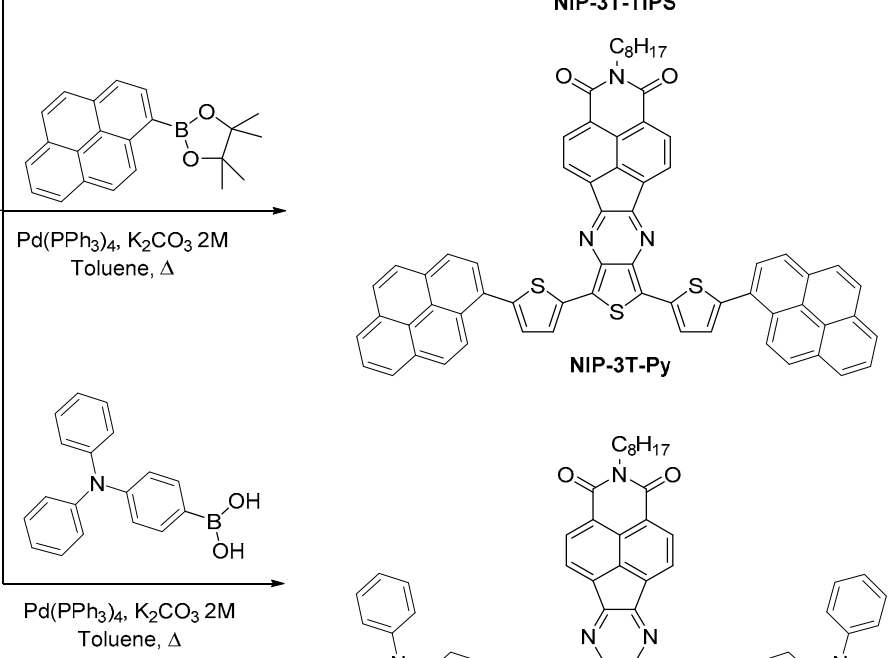

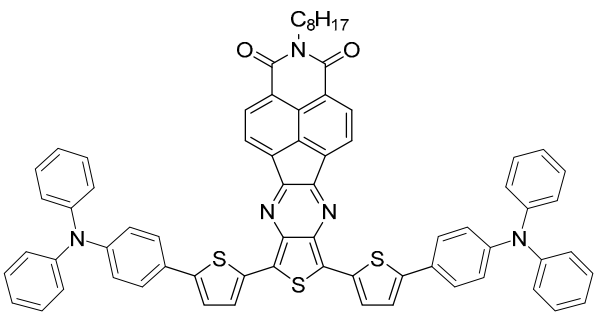

NIP-3T-TA

Scheme 8. Syntheses of D-A-D oligothiophene-naphthalimide assemblies. 


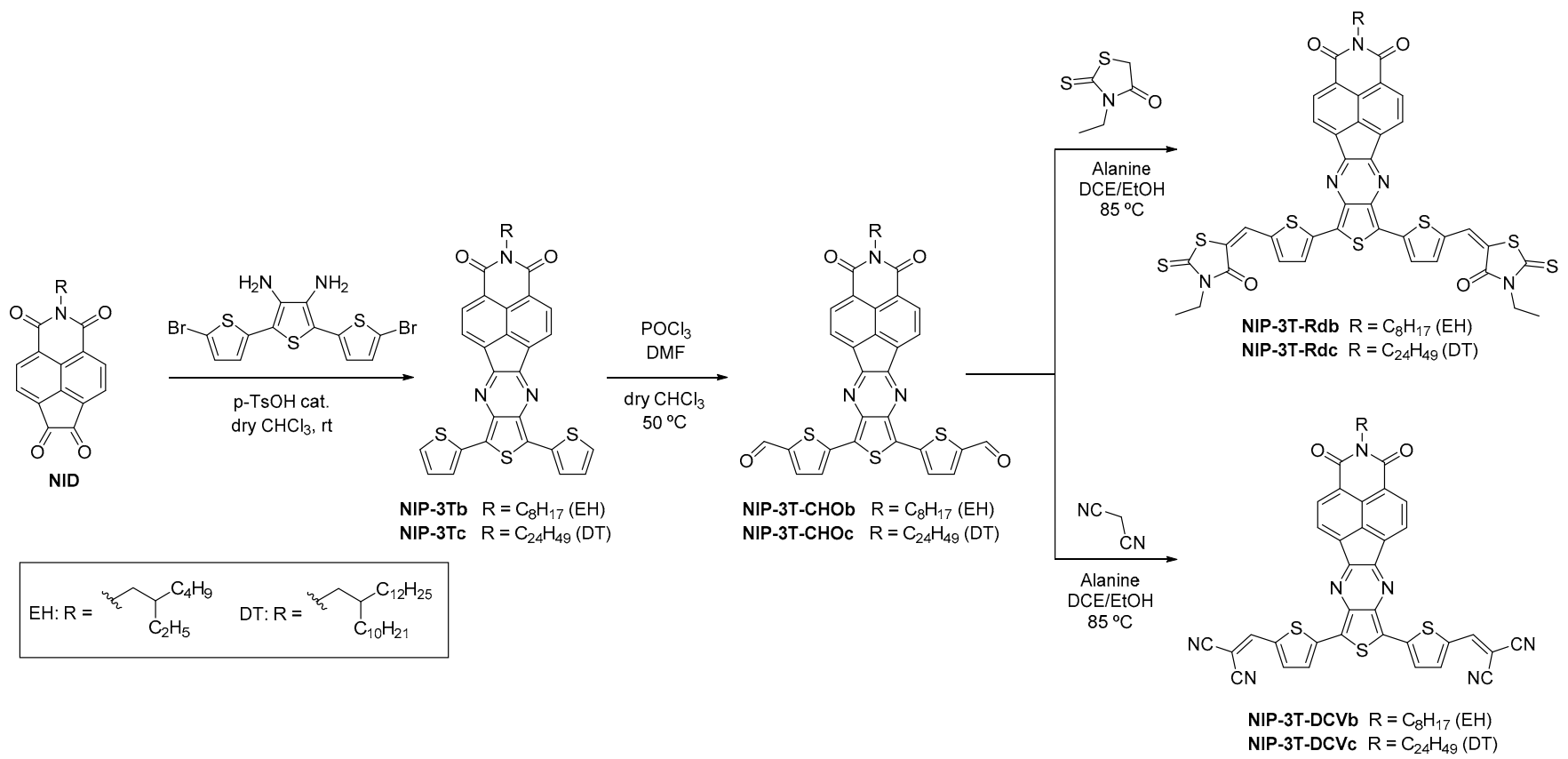

Scheme 9. Syntheses of A2-D-A1-D-A2 oligothiophene-naphthalimide assemblies.

The optimization of the most stable molecular structures for these thienopyrazinebased semiconductors shows completely planar structures for the central unit, as it was previously described for the NIP-3T core [28,29]. When nonbulky electron-acceptor groups are introduced in the molecular structure, such as in NIP-3T-Rd and NIP-3T-DCV, the assemblies remain coplanar and fully conjugated. In contrast, when bulky structures are introduced into the terminal positions of the oligothiophene, such as in NIP-3T-TA and NIP-3T-Py, they adopt noncoplanar configurations. Furthermore, to study the effect of the different substituents at the alpha positions of the thiophene units, DFT calculations were carried out to predict the most favorable dimeric conformations for these new assemblies. In the abovementioned studies [28,29], we demonstrated that for the NIP-3T core, the parallel and antiparallel dimeric conformations are quasi-isoenergetic. In contrast, when end-capped derivatives are introduced at the terminal positions, it can be observed that the most stable conformations are the parallel ones. In the case of NIP-3T-Rd and NIP3T-DCV, this behavior is due to the dipole moments of these molecules. For NIP-3T-Py, although the substituents are spatially out of the NIP-3T plane, close and cooperative $\pi-\pi$ interactions also promote the parallel stacking configuration (Figure 17).
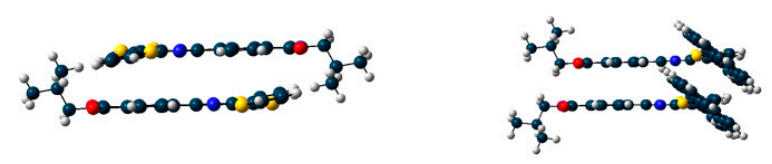

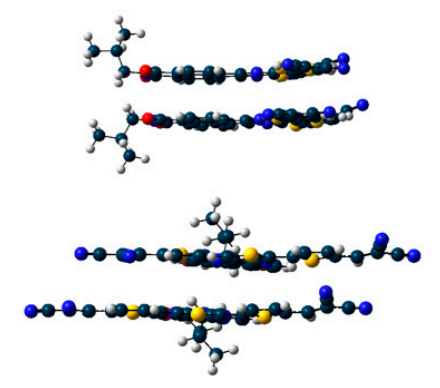

NIP-3T-DCV

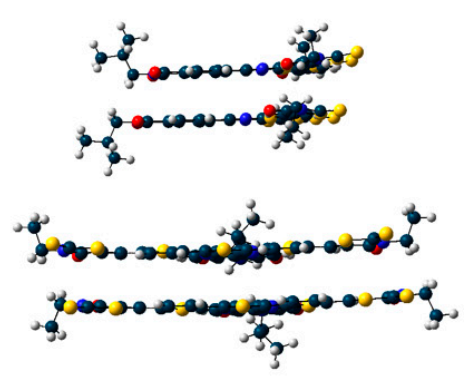

NIP-3T-Rd

Figure 17. Dimeric configuration calculated for the D-A-D and A2-D-A1-D-A2 semiconductors described in this section. Reproduced from [32] with permission from The Royal Society of Chemistry. 
The optical and electrochemical properties of the D-A-D and A2-D-A1-D-A2 assemblies have been compared to those of the NIP-3T building block (Tables 9 and 10). For NIP-3T-TA, NIP-3T-TIPS and NIP-3T-Py, the substitution with electron-rich units just redshifted the lowest energy band of the UV-vis absorption spectra. The decrease in the optical bad gap is mainly due to a destabilization of the HOMO levels, without significant modification of the LUMO energy level. In contrast, when the end-capped groups introduced at the terminal position of the oligothiophenes are strong electron acceptors, as in the case of NIP-3T-Rd and NIP-3T-DCV, the absorption spectra dramatically change. This substitution pattern allows for the formation of more extended $\pi$-conjugated systems, which is translated into an enhanced absorption cross section that extends through the whole visible range. Moreover, the topologies of the HOMO and LUMO levels of the A2-D-A1-D-A2 assemblies are different from those of the NIP-3T core. Thus, for the A2-DA1-D-A2 assemblies, frontier molecular orbitals are delocalized over the oligothiophene backbone (Figure 18), being more stabilized than in the unsubstituted analogue.

Table 9. Optical properties for the semiconductors based on end-capped thienopyrazine semiconductors measured in $\mathrm{CHCl}_{3}$ solution.

\begin{tabular}{|c|c|c|c|c|c|c|c|}
\hline Compound & {$[\mathrm{C}](\mu \mathrm{M})$} & $\lambda_{\max }(\mathrm{nm})$ & $\varepsilon_{\lambda \max }\left(\mathbf{M}^{-1} \mathrm{~cm}^{-1}\right)$ & $\lambda_{\varepsilon}(\mathrm{nm})$ & $\varepsilon_{\lambda \varepsilon}\left(\mathbf{M}^{-1} \mathrm{~cm}^{-1}\right)$ & $\lambda_{\text {ons }}(\mathrm{nm})$ & $E_{\mathrm{g}}$ opt a \\
\hline NIP-3T-TIPS & 0.100 & 349 & 167,000 & 576 & 48,000 & 689 & 1.79 \\
\hline NIP-3T-Py & 0.275 & 348 & 36,000 & 620 & 8360 & 760 & 1.63 \\
\hline NIP-3T-TA & 0.200 & 370 & 45,500 & 671 & 17,500 & 831 & 1.49 \\
\hline NIP-3Tb & 0.275 & 348 & 68,000 & 574. & 14,910 & 698 & 1.77 \\
\hline NIP-3T-Rdb & 0.275 & 353 & 38,180 & 712 & 17,820 & 774 & 1.60 \\
\hline NIP-3T-DCVb & 0.275 & 609 & 40,360 & 735 & 11,640 & 899 & 1.38 \\
\hline NIP-3Tc & 0.275 & 346 & 18,910 & 572 & 3636 & 694 & 1.79 \\
\hline NIP-3T-Rdc & 0.275 & 354 & 53,450 & 703 & 26,910 & 768 & 1.61 \\
\hline NIP-3T-DCVc & 0.275 & 607 & 43,270 & 650 & 36,730 & 704 & 1.76 \\
\hline
\end{tabular}

${ }^{a}$ Energy band gap derived from the low-energy absorption edge using the equation $1240 / \lambda_{\text {ons }}$.

Table 10. Electrochemical potentials versus SCE in $\mathrm{CH}_{2} \mathrm{Cl}_{2}$ (referenced to $\mathrm{Fc} / \mathrm{Fc}^{+}$couple) of functionalized NIP-3T derivatives and frontier molecular orbital energies estimated from $\mathrm{CV}$ data.

\begin{tabular}{|c|c|c|c|c|c|c|c|c|}
\hline Compound & $E_{\text {red1 }}$ & $E_{\text {red2 }}$ & $E_{\text {red3 }}$ & $E_{\mathrm{ox} 1}$ & $E_{\mathrm{ox2}}$ & LUMO $^{c}$ & HOMO $^{d}$ & $E_{\mathrm{g}} \mathrm{CV} \mathrm{e}$ \\
\hline NIP-3T-TIPS & -1.34 & -1.69 & - & $0.66^{\mathrm{a}}$ & - & -3.76 & -5.76 & 2.00 \\
\hline NIP-3T-Py & -1.16 & -1.68 & - & $0.42^{b}$ & - & -3.76 & -5.52 & 1.76 \\
\hline NIP-3T-TA & -1.22 & -1.47 & - & $0.29^{a}$ & 0.51 & -3.94 & -5.39 & 1.45 \\
\hline NIP-3Tb & -1.30 & -1.81 & - & $0.50^{b}$ & - & -3.80 & -5.60 & 1.80 \\
\hline NIP-3T-Rdb & -1.22 & -1.37 & -1.70 & $0.57^{\mathrm{b}}$ & 0.95 & -3.88 & -5.67 & 1.79 \\
\hline NIP-3T-DCVb & -1.03 & -1.31 & -1.72 & $0.93^{a}$ & - & -4.07 & -6.03 & 1.96 \\
\hline NIP-3Tc & -1.34 & -1.74 & - & $0.48^{b}$ & - & -3.71 & -5.61 & 1.90 \\
\hline NIP-3T-Rdc & -1.24 & -1.36 & -1.70 & $0.60^{b}$ & - & -3.86 & -5.76 & 1.90 \\
\hline NIP-3T-DCVc & -1.07 & -1.39 & -1.77 & $0.89^{a}$ & - & -4.01 & -5.99 & 1.98 \\
\hline
\end{tabular}

${ }^{a}$ Half-wave potential of the reversible wave. ${ }^{\mathrm{b}}$ Anodic peak potential of the irreversible wave. ${ }^{\mathrm{c}}$ LUMO level estimated from $E_{\mathrm{LUMO}}=-5.1$ $\mathrm{eV}-E_{\mathrm{red} 1} \cdot{ }^{\mathrm{d}} \mathrm{HOMO}$ level estimated from $E_{\mathrm{HOMO}}=-5.1 \mathrm{eV}-E_{\mathrm{ox} 1} \cdot{ }^{\mathrm{e}} E_{\mathrm{g}}{ }^{\mathrm{CV}}=E_{\mathrm{LUMO}}-E_{\mathrm{HOMO}}$. 


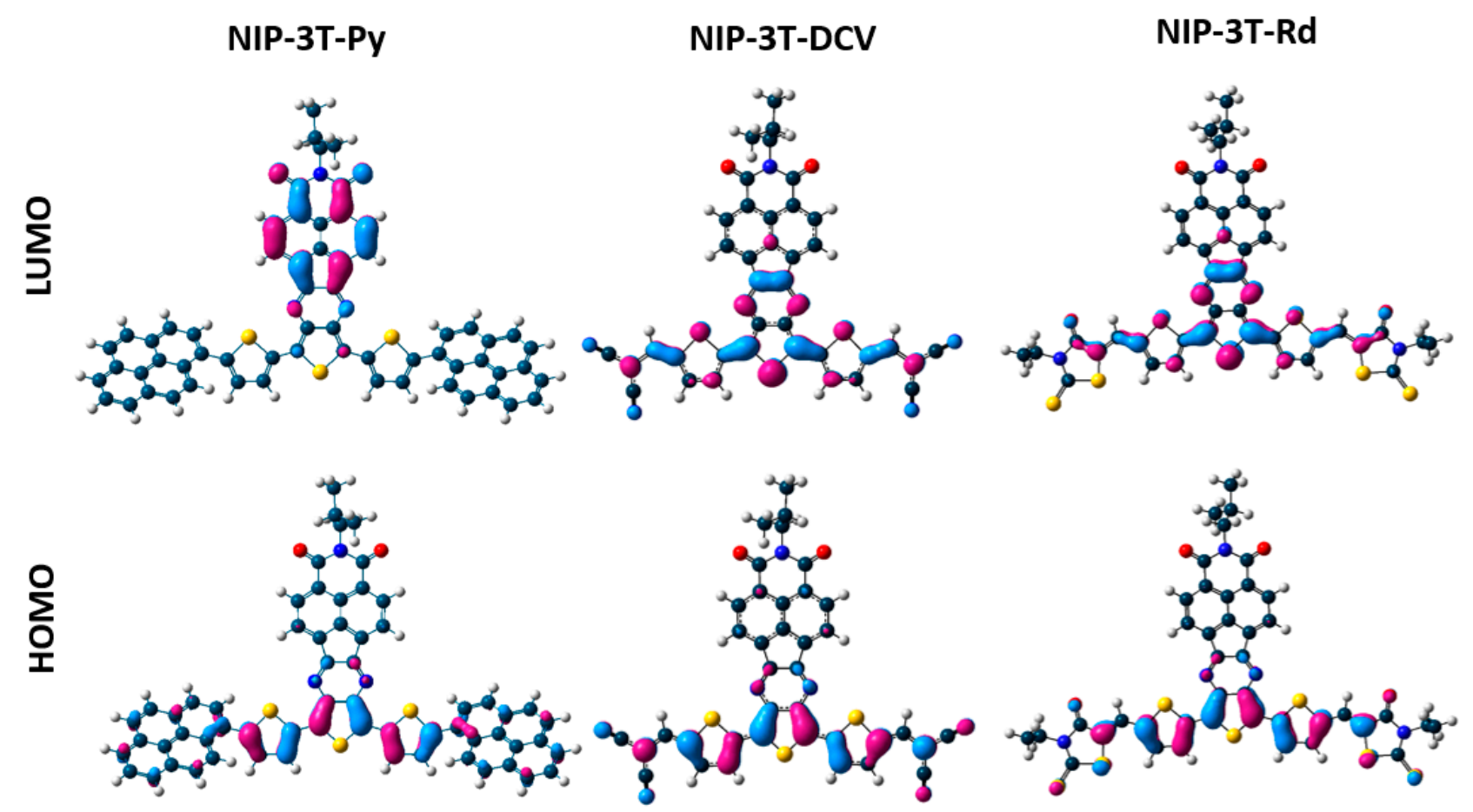

Figure 18. DFT/ / B3LYP/6-31G** molecular orbital topologies of NIP-3T, NIP-3T-Py, NIP-3T-DCV and NIP-3T-Rd. Adapted from [32] with permission from The Royal Society of Chemistry.

The electrical characterization of these new semiconductors was carried out in organic field-effect transistors with TC/BG architectures. The thin films deposited by vapor deposition were also characterized by XRD and AFM techniques. As previously shown, NIP-3Tb has ambipolar characteristics, with balanced holes and electron mobility values of $4 \times 10^{-5} \mathrm{~cm}^{2} \mathrm{~V}^{-1} \mathrm{~s}^{-1}$. The introduction of lateral substituents modifies the polarity in the charge transport properties, as it was shown for NIP-3T-TIPS, in which only $p$-type characteristics were found. On the other hand, when pyrene moieties are introduced laterally in the NIP-3T-Py derivative, electron mobilities of $10^{-2} \mathrm{~cm}^{2} \mathrm{~V}^{-1} \mathrm{~s}^{-1}$ were found, the best of all these semiconductors (Table 11). In contrast, when electron-withdrawing groups are introduced, only n-type mobilities were observed, except in the case of NIP3T-Rdb in which ambipolarity is observed (Table 11). The different behavior observed for the end-capped oligothiophene-naphthalimide semiconductors can be also related to the limited crystallinity observed in XRD and AFM images. It is worth pointing out that, given the suitable HOMO and LUMO energy levels, as well as good processability and full absorption range even in the NIR spectra of the oligothiophene-naphthalimide assemblies endowed with electron-acceptor units, they have also recently received some attention as nonfullerene acceptors in OSCs [33]. 
Table 11. OFET electrical data for vapor-deposited films of NIP-3Tb, NIP-3T-DCVb, NIP-3T-Rdb, NIP-3T-Py and NIP-3T-TIPS measured under vacuum on vapor-treated $\mathrm{Si} / \mathrm{SiO}_{2}$ substrates. Average field-effect mobilities are shown ${ }^{\mathrm{a}}$.

\begin{tabular}{|c|c|c|c|c|c|c|c|c|}
\hline Semiconductor & $S^{\mathbf{b}}$ & $T_{d}\left({ }^{\circ} \mathrm{C}\right)^{\mathrm{c}}$ & $\mu_{e}$ & $V_{T}$ & $\mathrm{I}_{\mathrm{ON}} / \mathrm{I}_{\mathrm{OFF}}$ & $\mu_{h}$ & $V_{T}$ & $\mathbf{I}_{\mathrm{ON}} / \mathrm{I}_{\mathrm{OFF}}$ \\
\hline NIP-3Tb & $\mathrm{H}$ & 90 & $4 \times 10^{-5}$ & 47 & $7 \times 10^{2}$ & $4 \times 10^{-5}$ & -47 & $3 \times 10^{3}$ \\
\hline \multirow{2}{*}{ NIP-3T-DCVb } & $\mathrm{O}$ & 90 & $2 \times 10^{-5}$ & 48 & $2 \times 10^{4}$ & - & - & - \\
\hline & $\mathrm{H}$ & 90 & $2 \times 10^{-5}$ & 54 & $2 \times 10^{6}$ & - & - & - \\
\hline \multirow{2}{*}{ NIP-3T-Rdb } & $\mathrm{O}$ & 90 & $1 \times 10^{-4}$ & 47 & $3 \times 10^{4}$ & $1 \times 10^{-5}$ & -50 & $1 \times 10^{2}$ \\
\hline & $\mathrm{H}$ & 90 & $1 \times 10^{-4}$ & 46 & $5 \times 10^{3}$ & $5 \times 10^{-6}$ & -15 & $3 \times 10^{1}$ \\
\hline \multirow{2}{*}{ NIP-3T-Py } & $\mathrm{H}$ & 90 & $4 \times 10^{-4}$ & 8 & $2 \times 10^{2}$ & - & - & - \\
\hline & $\mathrm{O}$ & 90 & $1 \times 10^{-2}$ & 42 & $9 \times 10^{2}$ & - & - & - \\
\hline NIP-3T-TIPS & $\mathrm{H}$ & 90 & - & - & - & $4 \times 10^{-5}$ & -31 & $2 \times 10^{1}$ \\
\hline
\end{tabular}

As it was previously mentioned, the implementation of conjugated molecular assemblies into polymeric materials has proven to be an efficient strategy to develop new semiconductors for a wide variety of applications [34-37]. Among them, the development of metal-free, organic polymers as photocatalysts [38] for the removal of hazardous contaminants $[39,40]$ has been revealed as an emerging area in the last decade.

In this regard, molecular- and polymeric-conjugated assemblies with wide absorption cross-section characteristics are good candidates as photocatalysts. Moreover, it is known that materials which combine electron donor and electron acceptor units are also interesting in photocatalysis because fast recombination processes may be inhibited. The possibilities offered by the oligothiophene-naphthalimide assemblies to efficiently tune their optical and electronic properties as well as their suitability to be functionalized at the $\alpha$ positions of the oligothiophene moieties make these types of assemblies good candidates to be used as monomers for the syntheses of donor-acceptor polymeric materials suitable for the photocatalytic degradation of organic pollutants in water [41].

As it is depicted in Scheme 10, the synthesis of the poly(azomethine) network NIP-3TANW is consummated by a Schiff-base condensation reaction between linear disubstituted oligothiophene-naphthalimide aldehyde NIP-3T-CHOb [33] with a trigonal monomer endowed with amine functional groups TAPB [42] under solvothermal reaction conditions [43]. Interestingly, this polymeric material shows high thermal stability, without noticeable weight loss up to $450{ }^{\circ} \mathrm{C}$, in contrast with the NIP-3T molecular semiconductor in which the degradation starts at $200{ }^{\circ} \mathrm{C}$. Regarding its optoelectronic properties, this material shows solid state absorption in the whole UV-vis spectrum, extending even up to $1200 \mathrm{~nm}$ in the NIR. In addition to its good light harvesting properties, an efficient electron-hole dissociation can be expected for NIP-3T-ANW due to the presence of the donor-acceptor units. Furthermore, the material exhibits a good energy band alignment for the photodegradation of some organic pollutants, such as rhodamine B $(\mathbf{R h B})$. Due to the above characteristics, the potential of NIP-3T-ANW as photocatalyst in the photodegradation of $\mathbf{R h B}$ has been investigated. It is known that RhB is stable in aqueous solution under illumination in the absence of catalyst. In contrast, when NIP-3T-ANW is added, after $120 \mathrm{~min}$, almost a $90 \%$ of $\mathbf{R h B}$ is degraded in the aqueous solution, and no stability problems in the catalyst are detected after four catalytic cycles. For comparison purposes, the photocatalytic activity of the analogue molecular component NIP-3T has been also investigated, showing only a $55 \%$ degradation of $\mathrm{RhB}$ in the same time period. Thus, the incorporation of the D-A unit into the polymeric network dramatically increases its activity and allows for its recyclability without losing efficiency, as shown in Figure 19. 


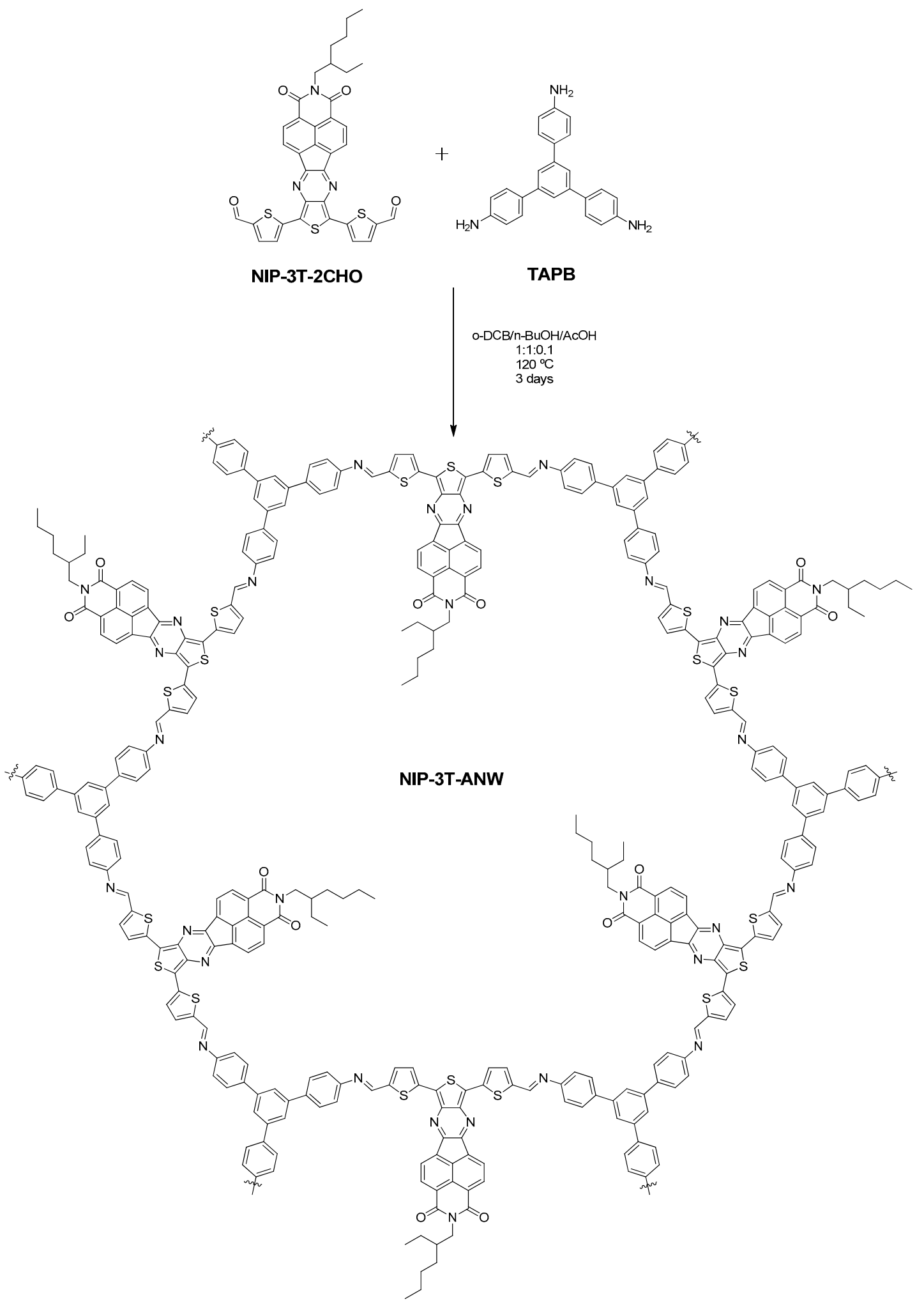

Scheme 10. Synthetic strategy for the obtention of NIP-3T-ANW semiconducting polymer. 


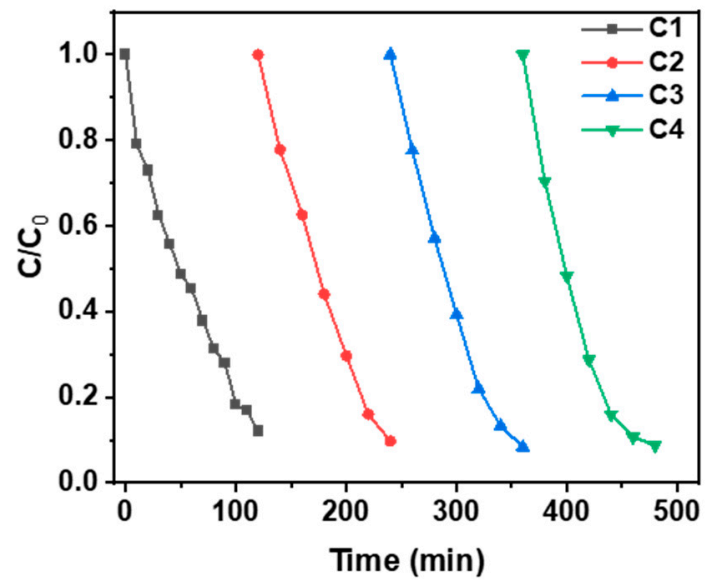

(a)

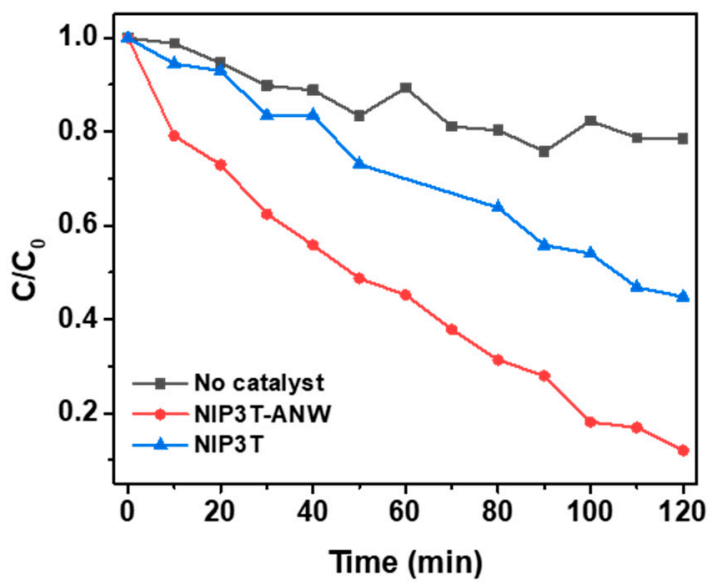

(b)

Figure 19. RhB degradation curves. (a) Comparison among NIP3T, NIP3T-ANW and without catalyst in the degradation performance. (b) Stability of NIP3T-ANW after four recycling cycles. Reproduced from M. J. Alonso-Navarro, J. Barrio, S. Royuela, N. Karjule, M. M. Ramos, J. I. Martínez, M. Shalom and J. L. Segura, RSC Adv. 2021, 11, 2701—Published by The Royal Society of Chemistry [41].

\section{Conclusions}

In this feature article, we have described and compared several families of oligothiophene-naphthalimide assemblies in which the donor and the acceptor units are connected through different rigid heterocycles. The synthetic modifications in the molecular structure, by growing the oligothiophene or the rylenimide moieties, is translated into a precise control over the frontier molecular orbital energy levels, allowing for the segregation of the HOMO and the LUMO in the molecule, promoting better charge carrier pathways. The structural modifications in these molecular systems produce changes in the microstructure domains, in the crystal diffraction patterns and in the electrical performance found in the thin-film organic field-effect transistors. Moreover, it has been shown that the modification of the naphthalimide-oligothiophene central core is not the only effective way to promote order into the molecule. In fact, the introduction of lateral hydrophilic or hydrophobic chains or end-capped terminal groups also represent interesting approaches to obtain organized assemblies via supramolecular interplay, such as Van der Waals interactions or $\pi-\pi$ stacking. Finally, the incorporation of these D-A assemblies into polymeric structures produces innovative materials with applications in different fields, including the photocatalytic degradation of organic pollutants in aqueous media.

We believe that the approaches outlined in this feature article concerning the precise modification of frontier molecular orbital energies and topologies in D-A assemblies based on oligothiophene-naphthalimide semiconductors can pave the way in the near future for the design and synthesis of more efficient materials for (opto)electronics and photocatalysis.

Author Contributions: Writing—original draft preparation, M.J.A.-N., E.G. and M.M.R.; Writingreview and editing, M.J.A.-N, E.G., M.M.R., R.P.O. and J.L.S.; Conceptualization, Project administration and Funding acquisition, J.L.S. and R.P.O. All authors have read and agreed to the published version of the manuscript.

Funding: This work was financially supported by MICINN (PID2019-106268GB-C33) and the UCM (INV.GR.00.1819.10759). R.P.O. acknowledges funding from MICINN (PID2019-110305GB-I00) and Junta de Andalucía (UMA18-FEDERJA-080 and P18-FR-4559).

Institutional Review Board Statement: Not applicable.

Informed Consent Statement: Not applicable. 
Data Availability Statement: The data underlying this article will be shared on reasonable request from the corresponding author

Acknowledgments: M.J.A.-N. gratefully acknowledges Universidad Rey Juan Carlos for a predoctoral contract.

Conflicts of Interest: The authors declare no conflict of interest. The funders had no role in the design of the study; in the collection, analyses, or interpretation of data; in the writing of the manuscript, or in the decision to publish the results.

\section{References}

1. Guo, X.; Facchetti, A.; Marks, T.J. Imide- and Amide-Functionalized Polymer Semiconductors. Chem. Rev. 2014, $114,8943-9021$. [CrossRef] [PubMed]

2. Sun, H.; Wang, L.; Wang, Y.; Guo, X. Imide-Functionalized Polymer Semiconductors. Chem. Eur. J. 2019, 25, 87-105. [CrossRef]

3. Paterson, A.F.; Singh, S.; Fallon, K.J.; Hodsden, T.; Han, Y.; Schroeder, B.C.; Bronstein, H.; Heeney, M.; McCulloch, I.; Anthopoulos, T.D. Recent Progress in High-Mobility Organic Transistors: A Reality Check. Adv. Mater. 2018, 30, 1801079. [CrossRef] [PubMed]

4. Yang, J.; Zhao, Z.; Wang, S.; Guo, Y.; Liu, Y. Insight into High-Performance Conjugated Polymers for Organic Field-Effect Transistors. Chem 2018, 4, 2748-2785. [CrossRef]

5. Sakai, N.; Mareda, J.; Vauthey, E.; Matile, S. Core-substituted naphthalenediimides. Chem. Commun. 2010, 46, 4225-4237. [CrossRef] [PubMed]

6. Zheng, N.; Lin, Z.; Zheng, Y.; Li, D.; Yang, J.; Zhang, W.; Wang, L.; Yu, G. Room-temperature stable organic spin valves using solution-processed ambipolar naphthalenediimide-based conjugated polymers. Org. Electron. 2020, 81, 105684. [CrossRef]

7. Shukla, J.; Mukhopadhyay, P. Synthesis of Functionalized Naphthalene Diimides and their Redox Properties. Eur. J. Org. Chem. 2019, 2019, 7770-7786. [CrossRef]

8. Zhan, X.; Facchetti, A.; Barlow, S.; Marks, T.J.; Ratner, M.A.; Wasielewski, M.R.; Marder, S.R. Rylene and Related Diimides for Organic Electronics. Adv. Mater. 2011, 23, 268-284. [CrossRef] [PubMed]

9. Guo, X.; Watson, M.D. Conjugated Polymers from Naphthalene Bisimide. Org. Lett. 2008, 10, 5333-5336. [CrossRef] [PubMed]

10. Blanco, R.; Gómez, R.; Seoane, C.; Segura, J.L.; Mena-Osteritz, E.; Bäuerle, P. An Ambipolar Peryleneamidine Monoimide-Fused Polythiophene with Narrow Band Gap. Org. Lett. 2007, 9, 2171-2174. [CrossRef]

11. González, S.R.; Casado, J.; Navarrete, J.T.L.; Blanco, R.; Segura, J.L. A $\beta$-Naphthaleneimide-Modified Terthiophene Exhibiting Charge Transfer and Polarization Through the Short Molecular Axis. Joint Spectroscopic and Theoretical Study. J. Phys. Chem. A 2008, 112, 6732-6740. [CrossRef] [PubMed]

12. Ortiz, R.P.; Herrera, H.; Blanco, R.; Huang, H.; Facchetti, A.; Marks, T.J.; Zheng, Y.; Segura, J.L. Organic n-Channel Field-Effect Transistors Based on Arylenediimide-Thiophene Derivatives. J. Am. Chem. Soc. 2010, 132, 8440-8452. [CrossRef]

13. Kan, B.; Chen, X.; Gao, K.; Zhang, M.; Lin, F.; Peng, X.; Liu, F.; Jen, A.K.Y. Asymmetrical side-chain engineering of small-molecule acceptors enable high-performance nonfullerene organic solar cells. Nano Energy 2020, 67, 104209. [CrossRef]

14. Welford, A.; Maniam, S.; Gann, E.; Jiao, X.; Thomsen, L.; Langford, S.J.; McNeill, C.R. Influence of alkyl side-chain type and length on the thin film microstructure and OFET performance of naphthalene diimide-based organic semiconductors. Org. Electron. 2019, 75, 105378. [CrossRef]

15. Hüttner, S.; Sommer, M.; Steiner, U.; Thelakkat, M. Organic field effect transistors from triarylamine side-chain polymers. Appl. Phys. Lett. 2010, 96, 073503. [CrossRef]

16. Huang, C.; Barlow, S.; Marder, S.R. Perylene-3,4,9,10-tetracarboxylic Acid Diimides: Synthesis, Physical Properties, and Use in Organic Electronics. J. Org. Chem. 2011, 76, 2386-2407. [CrossRef]

17. Yoon, M.-H.; DiBenedetto, S.A.; Russell, M.T.; Facchetti, A.; Marks, T.J. High-Performance n-Channel Carbonyl-Functionalized Quaterthiophene Semiconductors: Thin-Film Transistor Response and Majority Carrier Type Inversion via Simple Chemical Protection/Deprotection. Chem. Mater. 2007, 19, 4864-4881. [CrossRef]

18. Ortiz, R.P.; Casado, J.; Hernández, V.; Navarrete, J.T.L.; Letizia, J.A.; Ratner, M.A.; Facchetti, A.; Marks, T.J. Thiophene-Diazine Molecular Semiconductors: Synthesis, Structural, Electrochemical, Optical, and Electronic Structural Properties; Implementation in Organic Field-Effect Transistors. Chem. Eur. J. 2009, 15, 5023-5039. [CrossRef]

19. Otero, T.F.; Arias-Pardilla, J.; Herrera, H.; Segura, J.L.; Seoane, C. Electropolymerization of naphthaleneamidinemonoimidemodified poly(thiophene). Phys. Chem. Chem. Phys. 2011, 13, 16513-16515. [CrossRef]

20. Ortiz, R.P.; Herrera, H.; Seoane, C.; Segura, J.L.; Facchetti, A.; Marks, T.J. Rational Design of Ambipolar Organic Semiconductors: Is Core Planarity Central to Ambipolarity in Thiophene-Naphthalene Semiconductors? Chem. Eur. J. 2012, 18, 532-543. [CrossRef]

21. Intemann, J.J.; Yao, K.; Ding, F.; Xu, Y.; Xin, X.; Li, X.; Jen, A.K.-Y. Enhanced Performance of Organic Solar Cells with Increased End Group Dipole Moment in Indacenodithieno[3,2-b]thiophene-Based Molecules. Adv. Funct. Mater. 2015, 25, $4889-4897$. [CrossRef]

22. Sutton, C.; Risko, C.; Brédas, J.-L. Noncovalent Intermolecular Interactions in Organic Electronic Materials: Implications for the Molecular Packing vs Electronic Properties of Acenes. Chem. Mater. 2016, 28, 3-16. [CrossRef] 
23. Yun, J.H.; Park, S.; Heo, J.H.; Lee, H.-S.; Yoon, S.; Kang, J.; Im, S.H.; Kim, H.; Lee, W.; Kim, B.; et al. Enhancement of charge transport properties of small molecule semiconductors by controlling fluorine substitution and effects on photovoltaic properties of organic solar cells and perovskite solar cells. Chem. Sci. 2016, 7, 6649-6661. [CrossRef] [PubMed]

24. Riaño Carnerero, A.; López Espejo, G.; Mancheño Real, M.J.; Eckstein, B.; González-Cano, R.C.; Melkonyan, F.S.; Facchetti, A.; Marks, T.J.; Casado, J.; López Navarrete, J.T.; et al. Even and odd oligothiophene-bridged bis-naphthalimides for n-type and ambipolar organic field effect transistors. J. Mater. Chem. C 2017, 5, 9439-9450. [CrossRef]

25. de la Peña, A.; Arrechea-Marcos, I.; Mancheño, M.J.; Ruiz Delgado, M.C.; López Navarrete, J.T.; Segura, J.L.; Ponce Ortiz, R. Tuning of the Electronic Levels of Oligothiophene-Naphthalimide Assemblies by Chemical Modification. Chem. Eur. J. 2016, 22, 13643-13652. [CrossRef] [PubMed]

26. De la Peña Ruigómez, A. Polímeros orgánicos conjugados: desde sistemas lineales a redes orgánicas covalentes. PhD Thesis, Universidad Complutense de Madrid, Madrid, Spain, 2018.

27. Herrera, H.; de Echegaray, P.; Urdanpilleta, M.; Mancheño, M.J.; Mena-Osteritz, E.; Bäuerle, P.; Segura, J.L. Linear and star-shaped naphthalimide-fused pyrazinacenes. Chem. Commun. 2013, 49, 713-715. [CrossRef]

28. de Echegaray, P.; Mancheño, M.J.; Arrechea-Marcos, I.; Juárez, R.; López-Espejo, G.; López Navarrete, J.T.; Ramos, M.M.; Seoane, C.; Ortiz, R.P.; Segura, J.L. Synthesis of Perylene Imide Diones as Platforms for the Development of Pyrazine Based Organic Semiconductors. J. Org. Chem. 2016, 81, 11256-11267. [CrossRef]

29. Ponce Ortiz , R.; Herrera, H.; Mancheño, M.J.; Seoane, C.; Segura, J.L.; Mayorga Burrezo, P.; Casado, J.; López Navarrete, J.T.; Facchetti, A.; Marks, T.J. Molecular and Electronic-Structure Basis of the Ambipolar Behavior of Naphthalimide-Terthiophene Derivatives: Implementation in Organic Field-Effect Transistors. Chem. Eur. J. 2013, 19, 12458-12467. [CrossRef]

30. Arrechea-Marcos, I.; de Echegaray, P.; Mancheño, M.J.; Ruiz Delgado, M.C.; Ramos, M.M.; Quintana, J.A.; Villalvilla, J.M.; Díaz-García, M.A.; López Navarrete, J.T.; Ponce Ortiz, R.; et al. Molecular aggregation of naphthalimide organic semiconductors assisted by amphiphilic and lipophilic interactions: a joint theoretical and experimental study. Phys. Chem. Chem. Phys. 2017, 19, 6206-6215. [CrossRef]

31. Quintana, J.A.; Villalvilla, J.M.; de la Peña, A.; Segura, J.L.; Díaz-García, M.A. Electron Transport in a Water-Soluble LiquidCrystalline Perylene Bisimide. J. Phys. Chem. C 2014, 118, 26577-26583. [CrossRef]

32. Shirota, Y.; Kageyama, H. Charge Carrier Transporting Molecular Materials and Their Applications in Devices. Chem. Rev. 2007, 107, 953-1010. [CrossRef]

33. Alonso-Navarro, M.J.; Harbuzaru, A.; de Echegaray, P.; Arrechea-Marcos, I.; Harillo-Baños, A.; de la Peña, A.; Ramos, M.M.; López Navarrete, J.T.; Campoy-Quiles, M.; Ponce Ortiz, R.; et al. Effective interplay of donor and acceptor groups for tuning optoelectronic properties in oligothiophene-naphthalimide assemblies. J. Mater. Chem. C 2020, 8, 15277-15289. [CrossRef]

34. Wang, M.; Baek, P.; Akbarinejad, A.; Barker, D.; Travas-Sejdic, J. Conjugated polymers and composites for stretchable organic electronics. J. Mater. Chem. C 2019, 7, 5534-5552. [CrossRef]

35. Ledwon, P.; Ovsiannikova, D.; Jarosz, T.; Gogoc, S.; Nitschke, P.; Domagala, W. Insight into the properties and redox states of n-dopable conjugated polymers based on naphtalene diimide units. Electrochim. Acta 2019, 307, 525-535. [CrossRef]

36. Lee, J.; Shin, E.-S.; Kim, Y.-J.; Noh, Y.-Y.; Yang, C. Controlling the ambipolarity of thieno-benzo-isoindigo polymer-based transistors: the balance of face-on and edge-on populations. J. Mater. Chem. C 2020, 8, 296-302. [CrossRef]

37. Li, K.; Wang, L.; Chen, Z.; Yang, X.; Yu, Y.-X.; Zhang, W.-D.; Wang, Y.; Shi, Y.; Loh, K.P.; Xu, Q.-H. Photocatalytic Hydrogen Evolution under Ambient Conditions on Polymeric Carbon Nitride/Donor- $\pi$-Acceptor Organic Molecule Heterostructures. Adv. Funct. Mater. 2020, 30, 2005106. [CrossRef]

38. Zhang, G.; Lan, Z.-A.; Wang, X. Conjugated Polymers: Catalysts for Photocatalytic Hydrogen Evolution. Angew. Chem. Int. Ed. 2016, 55, 15712-15727. [CrossRef] [PubMed]

39. Chen, F.; An, W.; Liu, L.; Liang, Y.; Cui, W. Highly efficient removal of bisphenol A by a three-dimensional graphene hydrogelAgBr@rGO exhibiting adsorption/photocatalysis synergy. Appl. Catal. B Environ. 2017, 217, 65-80. [CrossRef]

40. Deng, Z.; Zhou, J.; Miao, L.; Liu, C.; Peng, Y.; Sun, L.; Tanemura, S. The emergence of solar thermal utilization: solar-driven steam generation. J. Mater. Chem. A 2017, 5, 7691-7709. [CrossRef]

41. Alonso-Navarro, M.J.; Barrio, J.; Royuela, S.; Karjule, N.; Ramos, M.M.; Martínez, J.I.; Shalom, M.; Segura, J.L. Photocatalytic degradation of organic pollutants through conjugated poly(azomethine) networks based on terthiophene-naphthalimide assemblies. RSC Adv. 2021, 11, 2701-2705. [CrossRef]

42. de la Peña Ruigómez, A.; Rodríguez-San-Miguel, D.; Stylianou, K.C.; Cavallini, M.; Gentili, D.; Liscio, F.; Milita, S.; Roscioni , O.M.; Ruiz-González, M.L.; Carbonell, C.; et al. Direct On-Surface Patterning of a Crystalline Laminar Covalent Organic Framework Synthesized at Room Temperature. Chem. Eur. J. 2015, 21, 10666-10670. [CrossRef] [PubMed]

43. Segura, J.L.; Mancheno, M.J.; Zamora, F. Covalent organic frameworks based on Schiff-base chemistry: synthesis, properties and potential applications. Chem. Soc. Rev. 2016, 45, 5635-5671. [CrossRef] [PubMed] 